\title{
Scalability of Quasi-Hysteretic FSM-Based Digitally Controlled Single-Inductor Dual-String Buck LED Driver to Multiple Strings
}

\author{
Albert T. L. Lee, Johnny K. O. Sin, Fellow, IEEE, and Philip C. H. Chan, Fellow, IEEE
}

\begin{abstract}
There has been growing interest in single-inductor multiple-output (SIMO) dc-dc converters due to its reduced cost and smaller form factor in comparison with using multiple singleoutput converters. An application for such a SIMO-based switching converter is to drive multiple LED strings in a multichannel LED display. This paper proposes a quasi-hysteretic finite-statemachine-based digitally controlled single-inductor dual-output buck switching LED driver operating in discontinuous conduction mode (DCM) and extends it to drive multiple outputs. Based on the time-multiplexing control scheme in DCM, a theoretical upper limit of the total number of outputs in a SIMO buck switching LED driver for various backlight LED current values can be derived analytically. The advantages of the proposed SIMO LED driver include reducing the controller design complexity by eliminating loop compensation, driving more LED strings without limited by the maximum LED current rating, performing digital dimming with no additional switches required, and optimization of local bus voltage to compensate for variability of LED forward voltage $V_{F}$ in each individual LED string with smaller power loss. Loosely binned LEDs with larger $V_{F}$ variation can, therefore, be used for reduced LED costs.
\end{abstract}

Index Terms-Boundary conduction mode (BCM), discontinuous conduction mode (DCM), finite-state machine (FSM), singleinductor dual-output (SIDO), single-inductor multiple-output (SIMO).

\section{A} $\mathrm{N}$ LED driver is essentially a current source (or sink) which maintains a constant current required for achieving the desired color and luminous flux from an array of LEDs. A number of highly efficient switching LED drivers have been reported in the literature and their primary objective is to achieve high power conversion efficiency [1]-[11]. Besides efficiency, another important consideration is the scalability of the existing single-inductor dual-output (SIDO) switching converter to drive multiple independent LED strings in a single-inductor multiple-output (SIMO) topology for reduced cost and smaller

Manuscript received November 7, 2012; revised January 22, 2013; accepted March 11, 2013. Recommended for publication by Associate Prof. J. M. Alonso.

A. T. L. Lee and J. K. O. Sin are with the Department of Electronic and Computer Engineering, The Hong Kong University of Science and Technology, Kowloon, Hong Kong (e-mail: alee@ust.hk; eesin@ust.hk).

P. C. H. Chan is with Hong Kong Polytechnic University, Hong Kong (e-mail: Philipch.Chan@inet.polyu.edu.hk).

Color versions of one or more of the figures in this paper are available online at http://ieeexplore.ieee.org.

Digital Object Identifier 10.1109/TPEL.2013.2253804 form factor [12]. However, in practice, only a finite number of outputs can be served by each LED driver.

The prior arts of SIMO switching converter use either one of two ways to distribute energy from a single power supply to multiple outputs with a single inductor, namely multiple energizing phases [13]-[20] and single energizing phase per switching cycle [21]. The former with time-multiplexing control leads to much better suppression of cross regulation because the outputs are decoupled in time. In this paper, a quasi-hysteretic finite-state machine (FSM)-based digital control scheme is employed in a SIDO buck LED driver consisting of two independent parallel strings operating in discontinuous conduction mode (DCM). The extension of this SIDO architecture to SIMO is formally investigated. The proposed SIMO-based switching buck LED driver enables separate control of the three primary colors (red, green, and blue), thereby offering more flexibility for color mixing. The rest of this paper is organized as follows. Section II introduces the proposed quasi-hysteretic FSM-based digital controller for a SIDO switching buck LED driver operating in DCM. Section III provides a theoretical analysis on the scalability of the proposed digital control scheme from SIDO to SIMO and suggests a general formula for determining the theoretical upper bound in the total number of outputs in SIMO. Section IV shows Cadence Spectre simulation results that are used to verify the theoretical model. Section $\mathrm{V}$ contains the experimental results for the proposed digitally controlled SIDO buck LED driver. Section VI concludes our research effort.

II. QUASI-HYSTERETIC FSM-BASED DigITAL CONTROL FOR SIDO LED DRIVER

A SIDO switching converter with time-multiplexing control scheme operating in DCM was first reported in [13]-[16]. With such kind of time-multiplexing control scheme, a SIDO converter can easily be extended to drive multiple outputs and it exhibits negligible cross regulation in DCM. A SIMO parallelstring LED driver operating in DCM has recently been reported [12]. It uses an analog-based controller with dominant pole compensation for stability, and time-multiplexing control in DCM is employed to suppress cross regulation among the LED strings. Unlike conventional pulse width modulation (PWM)based analog controllers, the proposed digital controller utilizing quasi-hysteretic control does not require any compensation circuits because of its inherent stability [22]-[24], hence simplifying the control loop design and reducing the component count and cost. Quasi-hysteretic control offers a good 


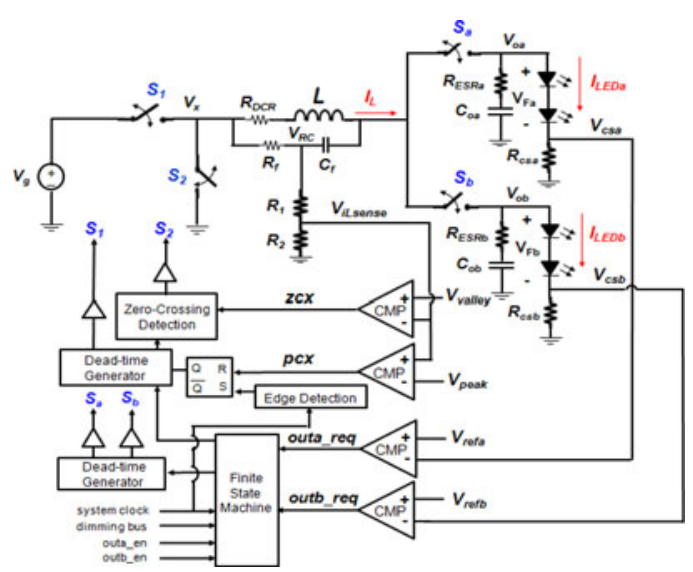

Fig. 1. System architecture of the quasi-hysteretic FSM-based digitally controlled SIDO buck LED driver.

compromise between traditional fixed-frequency PWM control and pure hysteretic control with variable switching frequency. In the proposed design, an external clock is used to synchronize the buck LED driver which switches at a fixed frequency. Fast comparators are used to control the on-time of the highside and low-side power switches by monitoring the inductor current. This is particularly suitable for lighting applications where variable switching frequencies are not desirable. The reconfigurability and scalability of a digital controller makes it especially attractive for SIMO.

A major drawback of the previously proposed SIMO LED driver operating in DCM [12] is that since the LED current is discontinuous, the LED endures a higher maximum peak current for the same average current required. In other words, the LED could potentially be operating close to its absolute maximum current rating, thereby increasing the current stress and possibly shortening the operating lifetime of the LED. In their approach, the LED current scales with the number of LED strings in SIMO. Hence, the maximum current rating of the LED unnecessarily restricts the maximum achievable number of LED strings which can be implemented in SIMO. In the proposed design, the LED current is always continuous and the LED can be regulated very close to the target average current value which is much lower than its maximum current rating. During the time interval when the output switch is OFF, the output capacitor, acting as a constant current source, continues to discharge its current to the corresponding LED string. When the output switch is ON, the power stage is reconnected to the LED string and the inductor current is transferred to the output capacitor and the LED string simultaneously. The current-sense feedback control ensures that the LED current is maintained at the desired dc level. Hence, a time-continuous current is supplied to the LED string. Consequently, the LED current does not scale with the number of LED strings in the proposed SIMO architecture, making it possible to drive more LED strings without inducing too much stress on the LEDs. Fig. 1 shows the system architecture of the proposed quasi-hysteretic FSM-based digitally controlled SIDO buck switching LED driver which takes into account the parasitic effects including the dc resistance (DCR) of the inductor $L$ and equivalent series resistance (ESR) of the output capacitors $\left(C_{o a}, C_{o b}\right)$. The two independently driven LED strings share the same inductor $L$ and the two main power switches $\left(S_{1}, S_{2}\right)$ of the buck converter. The output switches $\left(S_{a}, S_{b}\right)$ enable the charge stored in the inductor to be distributed between the two outputs in a time-multiplexed fashion. Dead-time generators are used to eliminate shoot-through current by ensuring that $S_{1}$ and $S_{2}$ are not turned ON simultaneously. Dead-times are also introduced between $S_{a}$ and $S_{b}$ to prevent inadvertent cross conduction between the two LED strings.

Since an LED is essentially a current driven device, an LED driver typically regulates the LED current rather than its forward voltage. A straightforward way is to insert a small high-precision current sensing resistor $\left(R_{\mathrm{csa}}, R_{\mathrm{csb}}\right)$ in series with the corresponding LED string to sense the LED current by converting it to the current-sense voltage $\left(V_{\mathrm{csa}}, V_{\mathrm{csb}}\right)$. The current-sense voltage is then compared with the reference voltage $\left(V_{\mathrm{refa}}, V_{\mathrm{refb}}\right)$ to generate the corresponding logic signals (outa_req, outb_req) which determine the opening or closing of the two output switches in a SIDO buck converter. Since the LED's $I-V$ curve is usually provided by the LED manufacturer, the target dc current value for a particular LED string can be set by choosing an appropriate reference voltage. On the other hand, a two-limit hysteretic control determines the on-time of the high-side and low-side power switches $\left(S_{1}, S_{2}\right)$ of the buck converter. The upper and lower limits of the inductor current, namely the peak current limit and the valley current limit, define the average value of the inductor current which is the total LED current for a SIDO buck LED driver. In DCM, the valley current limit is set to zero to prevent the inductor current from going negative which degrades the power conversion efficiency [12], [16], [25]. As illustrated in Fig. $1, R_{f} \mathrm{C}_{f}$ is connected in parallel to the inductor so that the slopes of $V_{R C}$ are proportional to the inductor current ramp-up and ramp-down slopes [26]. A small resistor ladder is connected between $V_{R C}$ and ground in order to generate a lower voltage signal $V_{i L \text { sense }}$ which falls within the input voltage range of the comparator (CMP). $V_{0 i L \text { sense }}$ is fed forward to the corresponding comparators to determine the peak-crossing and zero-crossing of the inductor current. Fig. 2 is a simplified flowchart showing the system-level operation of the proposed SIDO buck driver. Suppose identical current flows through each of the two LED strings, also referred to as the balanced load condition, the inductor current $I_{L}$ is assigned to each string in alternate switching cycles. The working principle of the proposed SIDO buck LED driver is represented by the timing diagram shown in Fig. 3. During $D_{1 a} T_{s}$ or $D_{1 b} T_{s}, I_{L}$ ramps up with a slope of $m_{1}=\left(V_{g}-V_{o}\right) / L$ and the inductor is charged with a voltage of $V_{L}=V_{g}-V_{o}$, where $V_{g}$ and $V_{o}$ represent the input voltage and the output voltage, respectively. During $D_{2 a} T_{s}$ or $D_{2 b} T_{s}, I_{L}$ ramps down with a slope of $m_{2}=$ $-V_{o} / L$ and the inductor discharges its current to the corresponding output capacitor and the LED string until $I_{L}$ returns to zero. During $D_{3 a} T_{s}$ or $D_{3 b} T_{s}, I_{L}$ stays at zero with both $S_{1}$ and $S_{2}$ OFF. In the proposed SIDO LED driver, the system clock defines the switching frequency. The rising edge of the system clock triggers the ON duty cycle $\left(D_{1 a} T_{s}, D_{1} b T_{s}\right)$ by charging up the inductor during which $S_{1}$ is ON and $S_{2}$ is OFF. The 


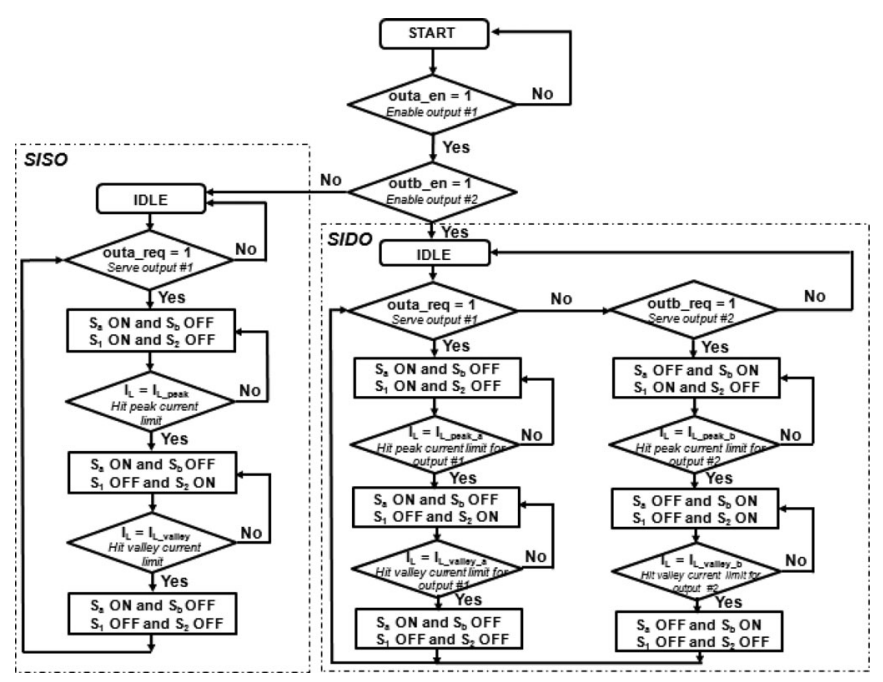

Fig. 2. Simplified flowchart representing the system-level operation of the proposed SIDO buck LED driver.

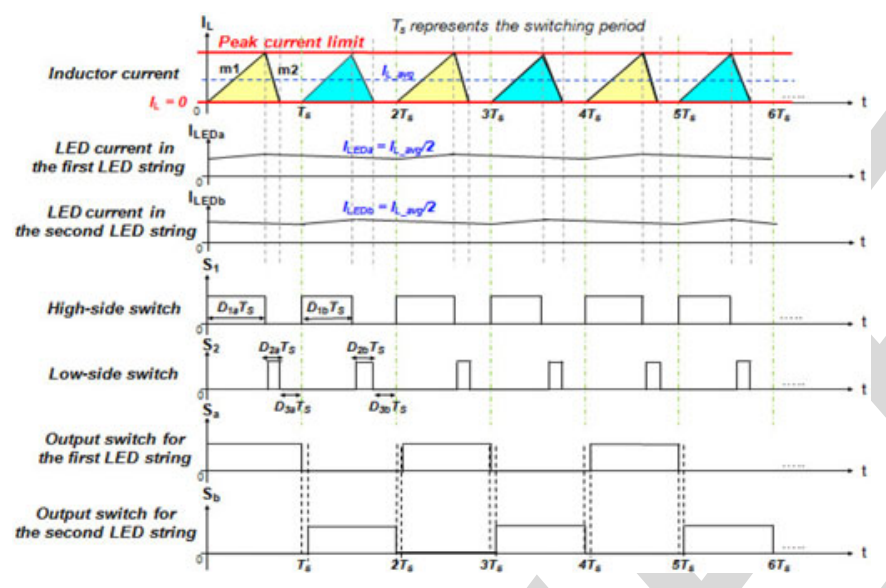

Fig. 3. Timing diagram of the proposed SIDO buck LED driver with balanced load operating in DCM. inductor current continues to increase until it hits the peak current limit at which point the buck converter enters $\left(D_{2 a} T_{s}, D_{2 b} T_{s}\right)$ where $S_{1}$ is OFF and $S_{2}$ is ON. The inductor discharges its current to the corresponding output until the zero-crossing of the inductor current is detected. The converter then enters the idle phase $\left(D_{3 a} T_{s}, D_{3 b} T_{s}\right)$ during which both $S_{1}$ and $S_{2}$ are OFF. The inductor current remains at zero until the next rising edge of the system clock arrives and the switching sequence repeats itself. The two output switches $\left(S_{a}, S_{b}\right)$ are controlled by the FSM as shown in Fig. 4.

The state machine is triggered by the rising edge of the system clock (sysclk) so that the transitions of the output switches $\left(S_{a}, S_{b}\right)$ are in sync with the system clock. The input signals of the state machine are the output enable signals (outa_en, outb_en) and the output request signals (outa_req, outb_req) which determine the switching sequence of the two outputs. The first LED string is always given a higher priority over the second one. For instance, if both strings request service simultaneously, i.e., outa_req $=1$ and $o u t b \_r e q=1, S_{a}$ is turned ON first and $S_{b}$ remains OFF. $S_{b}$ is turned ON only when outa_req $=0$

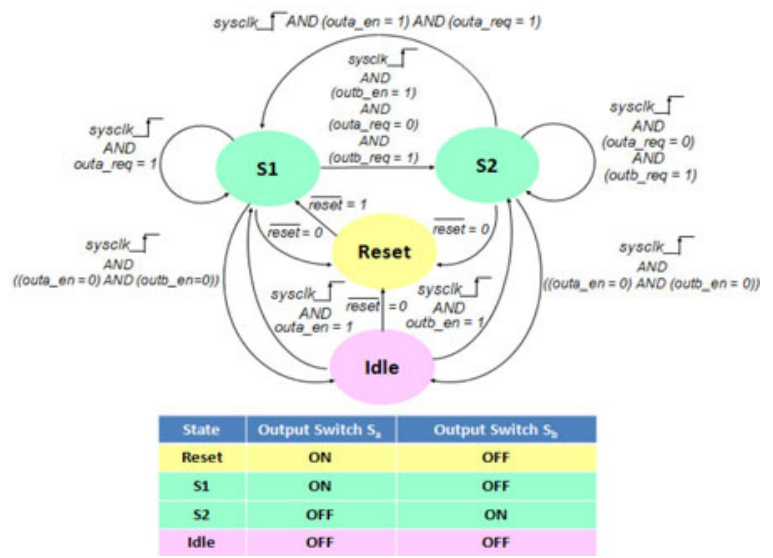

Fig. 4. State diagram of the proposed FSM for controlling the two output switches in SIDO buck LED driver.

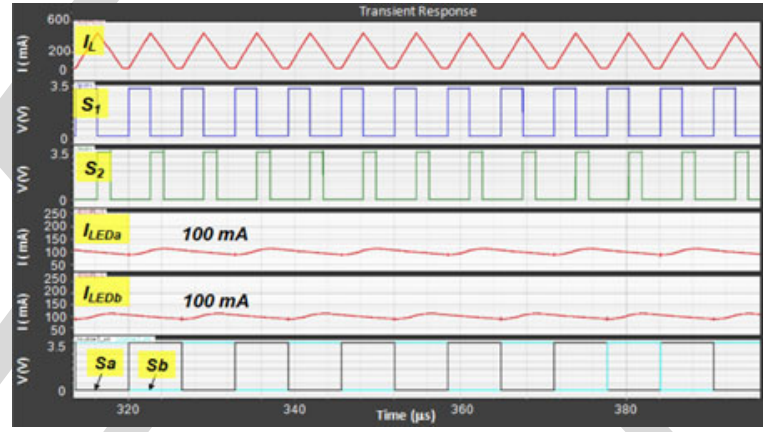

Fig. 5. Simulated steady-state waveforms for the proposed SIDO buck LED driver operating in DCM.

and $o u t b \_r e q=1 . S_{a}$ and $S_{b}$ must be nonoverlapping to avoid undesirable cross conduction between the two LED strings. In addition, an enable signal (outlen, out2en) is associated with either of the two LED strings. It provides the option of shutting down any or all of the LED strings, for example, in response to an overcurrent fault condition. An overriding signal can also be sent from the FSM to the hysteretic controller to disable the high-side and low-side power switches accordingly. The FSM-based controller can be modified quickly and conveniently to drive multiple LED strings in a SIMO configuration by simply adding more states in the VHDL or Verilog code. A mixed-signal macromodel of the proposed FSM-based digitally controlled SIDO buck switching LED operating in DCM is simulated in the time domain using Cadence Spectre [27]. The FSM is modeled in Verilog RTL and the rest are modeled as ideal circuit elements. The simulation model also incorporates parasitics such as DCR of the inductor $L$ and ESR of the output capacitors $\left(C_{o a}, C_{o b}\right)$. For balanced load condition, the current between the two LED strings is identical and each string consists of two LEDs connected in series. First, the steadystate performance is investigated. Fig. 5 contains the simulated steady-state waveforms for the inductor current $\left(I_{L}\right)$, the LED current $\left(I_{\mathrm{LED} a}, I_{\mathrm{LED} b}\right)$, and the four switches $\left(S_{1}, S_{2}, S_{a}, S_{b}\right)$ of the proposed SIDO buck LED driver operating in DCM. The switching frequency is $156.25 \mathrm{kHz}$ and the input voltage $V_{g}$ is $15 \mathrm{~V}$. The simulation results show that the LED current in either 


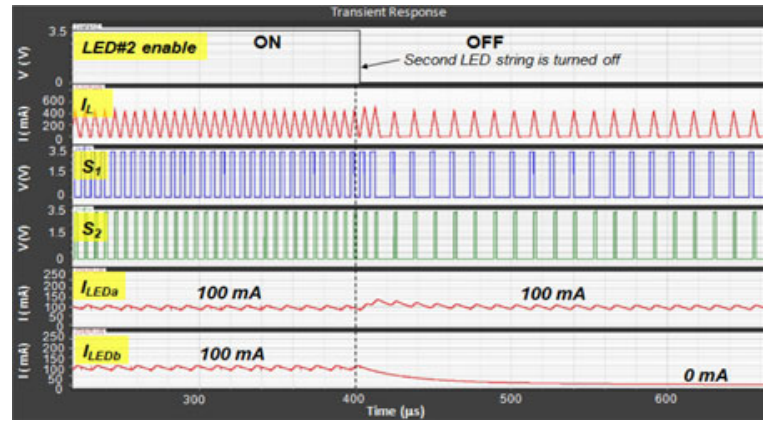

Fig. 6. First LED string remains under regulation without cross regulation when the second LED string is shut down completely.

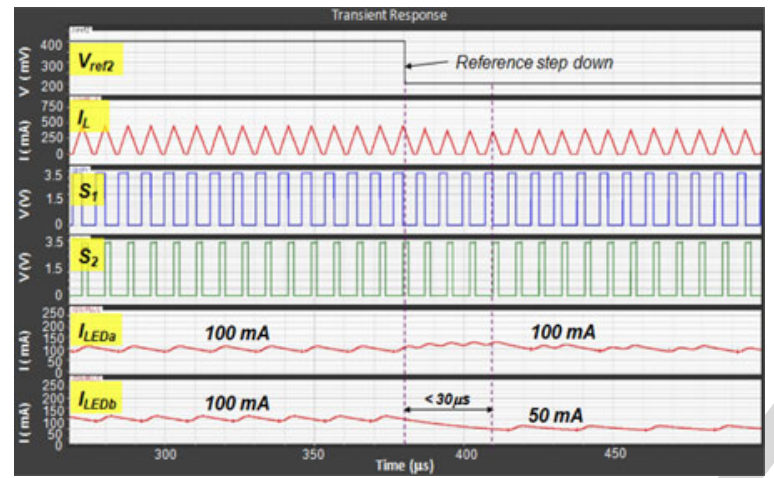

Fig. 7. First LED string remains under regulation without cross regulation despite a reference step in the second LED string from 100 to $50 \mathrm{~mA}$ in $20 \mathrm{~ns}$.

of the two strings is regulated successfully to the target steadystate dc value of $100 \mathrm{~mA}$ with a current ripple of $23 \backslash \%_{\mathrm{P}-\mathrm{P}}$. The steady-state output voltage for the first and second LED string is approximately $6.48 \mathrm{~V}$ with a voltage ripple of $2.6 \%$ P-P. Second, the stability of the closed-loop system is verified by examining its dynamic performance. In the first scenario, the second LED string needs to be shut down instantly in response to an over-current condition. Fig. 6 shows that despite the immediate shutdown of the second LED string, the LED current $I_{\mathrm{LED} a}$ in the first LED string continues to be regulated successfully at its target nominal value of $100 \mathrm{~mA}$ with minimal cross regulation. In the second scenario, the second LED string experiences a reference step of $50 \mathrm{~mA}$, i.e., $I_{\mathrm{LED} b}$ transitions from 100 to $50 \mathrm{~mA}$ in $20 \mathrm{~ns}$. Fig. 7 shows that the current in the first LED string continues to be regulated at around $100 \mathrm{~mA}$, virtually unaffected by the sudden reference step in the other string. The second LED string settles to the new nominal current value of $50 \mathrm{~mA}$. It demonstrates that the closed-loop system remains stable in response to the reference transient in the second string.

Unlike conventional backlight LED drivers that use PWM dimming transistor connected in series with the LED string [3], [8], [28]-[31], the proposed SIDO LED driver takes advantage of the existing four switches to perform dimming without requiring additional switches. When the dimming control signal for a particular LED string goes high, certain phases of the inductor current are skipped so that the average inductor current (also the average load current) going into that string is reduced accordingly. The digital dimming control signals (dim_ctrll,

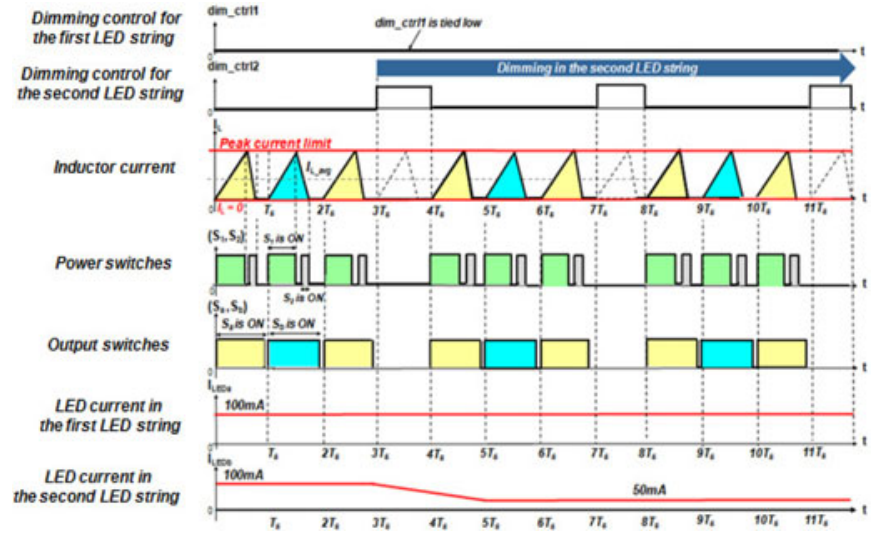

Fig. 8. Proposed digital dimming control in SIDO buck LED driver.

dim_ctrl2) essentially modulate the dc current level flowing 255 through the corresponding LED string. No additional dimming 256 transistors in series with the LED string are required, thereby 257 leading to a smaller voltage headroom and reduced power loss. 258 The only voltage headroom is the voltage across the current- 259 sense resistor $\left(V_{\text {csa }}, V_{\text {csb }}\right)$ which is typically between 0.2 and 260 $0.4 \mathrm{~V}$. Fig. 8 depicts the timing diagram of the proposed digital 261 dimming control scheme. In this particular case, the second LED 262 string is dimmed by reducing its current from 100 to $50 \mathrm{~mA}, 263$ while the current in the first LED string stays constant at $100 \mathrm{~mA} .264$

Any combination of LED strings in a SIMO LED driver can 265 be dimmed or even shut down momentarily to achieve flexi- 266 ble dimming and optimum luminance levels. In addition, it is 267 reported in the literature [32]-[34] that a bilevel or $N$-level cur- 268 rent driving technique for LED dimming improves the luminous 269 efficacy of LEDs by introducing a dc offset to the PWM cur- 270 rent. The proposed SIDO converter can potentially be used as 271 a bilevel LED driver by generating two programmable dc cur- 272 rent values for each individual LED string in a time-multiplexed 273 fashion. Another major difference between the proposed LED 274 driver and the existing ones [3], [8], [28]-[31] is that the former 275 provides $N$ optimized output bus voltage for each individual 276 LED string, whereas the latter only uses a common output bus 277 shared by all the LED strings. Due to manufacture, process, 278 and temperature variations, $V_{F}$ in each LED does not match 279 perfectly, which means that the voltage drop across each LED 280 string differs. Using the proposed SIDO buck LED driver in 281 Fig. 1 as an example and assuming the LED current is $100 \mathrm{~mA} 282$ in each string, the voltage headroom $\left(V_{\mathrm{csa}}, V_{\mathrm{csb}}\right)$ is $0.4 \mathrm{~V}$, and 283 the voltage drop across each of the two LED strings are $V_{F a}=284$ $6.0 \mathrm{~V}$ and $V_{F b}=7.0 \mathrm{~V}$, respectively. The output voltages are 285 $V_{o a}=6.4 \mathrm{~V}$ and $V_{o b}=7.4 \mathrm{~V}$. The total power consumption 286 of the load $P_{\mathrm{LOAD}}$, including the LED string and current-sense 287 resistor, is $P_{\mathrm{LOAD}}=V_{o a} \times I_{\mathrm{LED}}+V_{o b} \times I_{\mathrm{LED}}=1.38 \mathrm{~W}$. The 288 output voltage for each LED string is independently optimized 289 based on its corresponding $V_{F}$, resulting in the same voltage 290 headroom of $0.4 \mathrm{~V}$ for each string. This is different from a con- 291 ventional LED driver in which the common output bus voltage 292 is usually regulated using the LED string with the maximum 293 voltage drop. For the same LED current, the total power con- 294 sumption using a conventional LED driver is given by: $P_{\mathrm{LOAD}}=295$ 


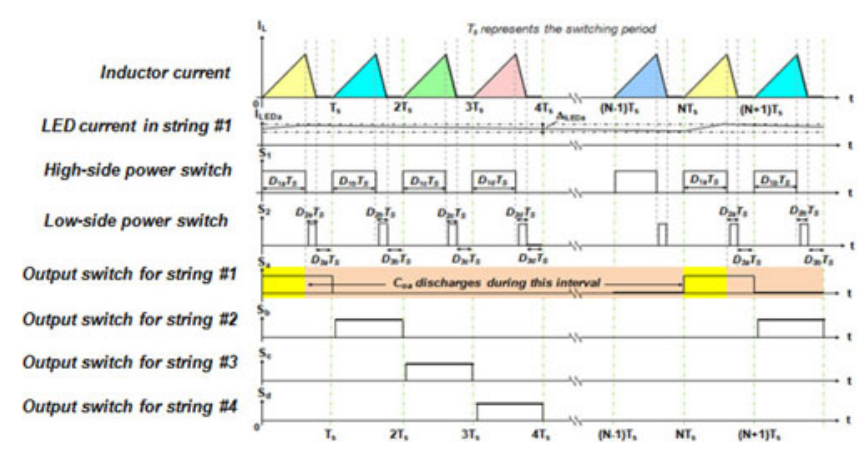

Fig. 9. Timing diagram of the proposed SIMO buck LED driver.

$2 \times \max \left(V_{o a}, V_{o b}\right) \times I_{\mathrm{LED}}=1.48 \mathrm{~W}$, which is more than $7 \%$ higher than that of the proposed driver. The voltage headroom for the first LED string increases from 0.4 to $1.4 \mathrm{~V}$, resulting in $450 \mathrm{~mW}$ more power loss or additional $30 \%$ efficiency degradation. Since the output voltage is self-optimized to match the total $V_{F}$ in each individual LED string in the proposed driver, same-colored LEDs from neighboring bins (not only from a single bin) with larger $V_{F}$ variance can be used which helps reduce the LED costs. In the event that a particular application demands a total LED current greater than the average inductor current, the same time-multiplexing control scheme operating in DCM can still be employed either by lowering the switching frequency with a higher inductor peak current limit or by operating the buck LED driver in pseudocontinuous conduction mode (PCCM) [17], [35], [36]. In PCCM, the average inductor current is increased by simply adding a nonzero dc offset of $I_{\mathrm{DC}}$ to that of DCM.

\section{EXTENSION FROM SIDO TO SIMO BUCK LED DRIVER}

Having demonstrated the feasibility of the proposed SIDO buck LED driver, it is natural for us to extend it to SIMO with $N$ independently driven LED strings. In particular, the theoretical maximum number of LED strings $N_{\max }$ is determined for this SIMO architecture. Fig. 9 shows a timing diagram of the inductor current, the two power switches $\left(S_{1}, S_{2}\right)$, and the first four output switches $\left(S_{a}, S_{b}, S_{c}, S_{d}\right)$ in a SIMO buck LED driver. To simplify the analysis, the balanced load condition is assumed. Based on the time-multiplexing control scheme, energy is being transferred from the dc supply to each individual output exactly once within a total of $N$ switching phases. For a particular output, the corresponding output switch is OFF during $D_{3}$, while the output capacitor discharges to the LED string. During the subsequent $(N-1) \times T_{s}$ phases, the output switch remains OFF and the output capacitor continues to discharge to the corresponding LED string. Hence, the total discharging time for the output capacitor $t_{\mathrm{dch}}$ can be expressed as

$$
\begin{aligned}
t_{\mathrm{dch}} & =D_{3} T_{s}+(N-1) T_{s}=\left(D_{3}+N-1\right) T_{s} . \\
\text { For } D_{3} & =0, t_{\mathrm{dch}}=(N-1) T_{s} .
\end{aligned}
$$

The proposed SIMO buck LED driver is essentially a constant-current regulator which maintains a constant dc current $I_{\mathrm{LED}}$ flowing through the LED string via a closed-loop current-sense feedback control. For very small variation of forward voltage around the quiescent point (also known as bias point) on the LED's exponential $I-V$ curve, the dc forward current is assumed to be constant. During $t_{\mathrm{dch}}$ when the output switch is OFF, the output capacitor is connected to the LED string which acts as a constant-current sink (CCS). Assuming ideal capacitor with no ESR (the effect of the ESR will be explained later), the voltage across the output capacitor $v_{c}(\mathrm{t})$ is the same as the output voltage which is expressed as the charge $q(t)$ divided by the capacitance value $C_{o}$, i.e.,

$$
v_{c}(t)=\frac{q(t)}{C_{o}}=\frac{1}{C_{o}} \int_{0}^{t_{\mathrm{dch}}} i_{c}(\tau) d \tau+v_{c}(0)
$$

For CCS, $i_{c}(\tau)=I_{\mathrm{LED}}$.

Combining (3) and (4) and rearranging, we have

$$
\Delta v_{o}=\Delta v_{c}=v_{c}(t)-v_{c}(0)=\frac{1}{C_{o}}\left(I_{\mathrm{LED}} t_{\mathrm{dch}}\right) .
$$

Hence, the total discharging time $t_{\mathrm{dch}}$ can be expressed as

$$
t_{\mathrm{dch}}=\frac{C_{o} \Delta v_{o}}{I_{\mathrm{LED}}}
$$

where $\Delta v_{o}$ is the output voltage drop due to the discharging 346 of the output capacitor. In general, $\Delta v_{o}$ is assumed to be rea- 347 sonably small relative to the output voltage. The LED ripple 348 current $\Delta i_{\text {LED }}$ usually ranges from $10 \%$ P-P to $40 \%$ P-P of the 349 dc forward current as recommended by the LED manufactur- 350 ers [37], [38]. For a particular $\Delta i_{\mathrm{LED}}$, the corresponding voltage 351 ripple $\Delta v_{\mathrm{LED}}$ at the chosen bias point can be readily obtained 352 from the exponential $I-V$ curve. Suppose each LED string con- 353 tains a total of $n$ LEDs connected in series. The output voltage 354 ripple $\Delta v_{o}$ is, therefore, the sum of the voltage ripple across 355 the LED string and the voltage ripple across the current-sense 356 resistor, i.e., $\Delta v_{o}=n \times \Delta v_{\mathrm{LED}}+\Delta v_{c s}$. Suppose $\Delta v_{\text {omax }}$ rep- 357 resents the maximum output voltage ripple allowed. Equation (6) 358 can, therefore, be rewritten as

$$
t_{\mathrm{dch}} \leq \frac{C_{o} \Delta v_{o \text { max }}}{I_{\mathrm{LED}}} .
$$

Substituting (1) into (7), we have

$$
\begin{aligned}
\left(D_{3}+N-1\right) T_{s} & \leq \frac{C_{o} \Delta v_{o \text { max }}}{I_{\mathrm{LED}}} \Rightarrow N \\
& \leq \frac{C_{o} \Delta v_{o \text { max }}}{I_{\mathrm{LED}} T_{s}}+1-D_{3} .
\end{aligned}
$$

Hence, the theoretical maximum possible number of LED 361 strings in SIMO, $N_{\max }$, is given by

$$
N_{\max }=\frac{C_{o} \Delta v_{o \text { max }}}{I_{\mathrm{LED}} T_{s}}+1-D_{3}=\frac{C_{o} \Delta v_{o \text { max }}}{I_{\mathrm{LED}} T_{s}}+D_{1}+D_{2} .
$$

Since $N_{\max }$ is an integer value, the $\operatorname{floor}(\cdot)$ function is used to round the result down to the closest integer. Hence, (9a) becomes

$$
N_{\max }=\text { floor }\left(\frac{C_{o} \Delta v_{o \text { max }}+I_{\mathrm{LED}} T_{s}\left(1-D_{3}\right)}{I_{\mathrm{LED}} T_{s}}\right) .
$$

Equation (9b) represents a general formula for determining the 365 scalability limit of a SIMO buck LED driver operating in DCM 366

.

.

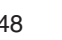

49

350

351

352

53

54

56


and is referred to as a scalable DCM-based SIMO scheme for the sake of our ensuing discussion. In particular, when $D_{3}=$ 0 , the SIMO buck LED driver operates in boundary conduction mode (BCM). Hence, (9a) and (9b) become (10a) and (10b), respectively. Also, $N_{\max }$ in $\mathrm{BCM}$ is greater than or equal to that in DCM for the same set of design parameter values

$$
\begin{aligned}
& N_{\text {max }}=\frac{C_{o} \Delta v_{o \text { max }}}{I_{\mathrm{LED}} T_{s}}+1 \\
& N_{\max }=\text { floor }\left(\frac{C_{o} \Delta v_{o \text { max }}}{I_{\mathrm{LED}} T_{s}}+1\right) .
\end{aligned}
$$

For a single-output buck converter, the average inductor current is identical to the load current. Due to the nature of the timemultiplexing control scheme in the proposed SIMO converter, the average inductor current $I_{L_{-a v g}}$ is the sum of the individual load current $I_{\mathrm{LED}}$ in each LED string. Assuming balanced load condition, $I_{L_{-} \text {avg }}=N \times I_{\mathrm{LED}}$, where $N$ is the total number of LED strings. The average inductor current reaches its maximum value in BCM, resulting in a maximum transfer of power [16]. Since the current in each LED string remains the same, a theoretical upper bound of the total achievable number of LED strings in SIMO can be expressed as

$$
N_{\max }=\frac{I_{L \_ \text {avg_max }}}{I_{\mathrm{LED}}} .
$$

By simple geometry, $I_{L_{-} \text {avg_max }}$ is given by the following equation [39]:

$$
I_{L_{\_} \text {avg_max }}=\frac{m_{1} D_{1} T_{s}}{2}=\frac{\left(V_{g}-V_{o}\right) D_{1} T_{s}}{2 L} .
$$

By substituting (12) into (11) and rearranging, $T_{s}$ can be expressed as

$$
T_{s}=\frac{2 L N_{\max } I_{\mathrm{LED}}}{D_{1}\left(V_{g}-V_{o}\right)} .
$$

Now, by substituting (13) into (10a) and rearranging, we have $2 L I_{\mathrm{LED}}^{2} N_{\mathrm{max}}^{2}-2 L I_{\mathrm{LED}}^{2} N_{\max }-C_{o} \Delta v_{o \text { max }}\left(V_{g}-V_{o}\right) D_{1}=0$.

$\Delta$ of (14) can be expressed as

$$
\Delta=4 L^{2} I_{\mathrm{LED}}^{4}+8 L I_{\mathrm{LED}}^{2} C_{o} \Delta v_{o \max }\left(V_{g}-V_{o}\right) D_{1}>0 .
$$

Since $\left(V_{g}-V_{o}\right)>0$ for a buck switcher, the discriminant in (15) is always a positive number which implies that (14) has two real roots as given by

$$
r_{1}, r_{2}=\frac{2 L I_{\mathrm{LED}}^{2} \pm \sqrt{\Delta}}{4 L I_{\mathrm{LED}}^{2}} .
$$

Since $N_{\max }$ must be a positive integer, the negative root is eliminated, leaving only the positive root, i.e.,

$$
\begin{aligned}
& N_{\max \_ \text {BCM }} \\
& =\text { floor }\left(\frac{1}{2} \times\left[1+\sqrt{1+\frac{2 C_{o} \Delta v_{o \text { max }} V_{o}\left(V_{g}-V_{o}\right)}{L I_{\mathrm{LED}}^{2} V_{g}}}\right]\right)
\end{aligned}
$$

Equation (17) defines the theoretical maximum total number of outputs in SIMO operating in BCM. It is referred to as a scalable BCM-based SIMO scheme which is a special case of 398 scalable DCM-based SIMO scheme. In fact, it is observed that (11) is also valid for the case of DCM. By simple geometry, the switching period $T_{s}$ in DCM can be expressed as

$$
T_{s}=\frac{2 L N_{\max } I_{\mathrm{LED}}}{D_{1}\left(D_{1}+D_{2}\right)\left(V_{g}-V_{o}\right)} .
$$

Realizing that the same calculations that lead to (17) for the 402 case of BCM can also be performed in DCM, the theoretical 403 maximum total number of LED strings in a SIMO converter 404 operating in DCM can, therefore, be written as ${ }^{1}$

$$
\begin{aligned}
& N_{\text {max _DCM }}=\text { floor }\left(\frac{1}{2} \times\left(1-D_{3}\right)\right. \\
&\left.\times\left[1+\sqrt{1+\frac{2 C_{o} \Delta v_{o \max }\left(V_{g}-V_{o}\right) D_{1}}{L I_{\mathrm{LED}}^{2}\left(1-D_{3}\right)}}\right]\right) .
\end{aligned}
$$

Notice that for the case of BCM, $D_{3}=0$ and $D_{1}=V_{o} / V_{g}, 406$ (19) reduces to (17). Hence, (19) represents the generalized 407 formula for the theoretical maximum total number of outputs 408 in SIMO which is applicable to either BCM or DCM. It is also 409 interesting to note that the average inductor current in DCM 410 is smaller than (or equal to) that in BCM. As a result, for the 411 same LED current, the theoretical maximum achievable number 412 of outputs in SIMO operating in DCM is no greater than that 413 in BCM, i.e., $N_{\max \_ \text {DCM }} \leq N_{\text {max_BCM }}$. In reality, the ESR of 414 the output capacitor needs to be taken into consideration. Any 415 current flowing through the output capacitor $C_{o}$ must also flow 416 through the $R_{\mathrm{ESR}}$, resulting in an additional voltage drop of 417 $\Delta V_{\mathrm{ESR}}=I_{\mathrm{LED}} \times R_{\mathrm{ESR}}$. Hence, $\Delta v_{o}$ can be expressed as

$$
\Delta v_{o}=\Delta v_{c}+\Delta v_{\mathrm{ESR}}=\Delta v_{c}+I_{\mathrm{LED}} \times R_{\mathrm{ESR}} .
$$

Rearranging the terms in (20), we have

$$
\Delta v_{c}=\Delta v_{o}-I_{\mathrm{LED}} \times R_{\mathrm{ESR}} .
$$

Hence, (17) and (19) are modified slightly to become (22) and 420 (23), respectively: BCM:

$$
\begin{gathered}
N_{\text {max } \_ \text {BCM }}=\text { floor }\left(\frac{1}{2} \times[1\right. \\
\left.+\sqrt{\left.1+\frac{2 C_{o} V_{o}\left(\Delta v_{o \text { max }}-I_{\mathrm{LED}} R_{\mathrm{ESR}}\right)\left(V_{g}-V_{o}\right)}{L I_{\mathrm{LED}}^{2} V_{g}}\right]}\right)
\end{gathered}
$$

DCM:

$$
\begin{aligned}
& N_{\text {max _DCM }}=\text { floor }\left(\frac{1}{2} \times\left(1-D_{3}\right) \times[1\right. \\
& \left.\left.+\sqrt{1+\frac{2 C_{o}\left(\Delta v_{o \text { max }}-I_{\mathrm{LED}} R_{\mathrm{ESR}}\right)\left(V_{g}-V_{o}\right) D_{1}}{L I_{\mathrm{LED}}^{2}\left(1-D_{3}\right)}}\right]\right) .
\end{aligned}
$$

The presence of $R_{\mathrm{ESR}}$ in (22) and (23) reduces the theoretical 423 maximum achievable number of outputs in SIMO. Therefore, 424

${ }^{1}$ In DCM, $D_{1}$ can be expressed as: $D_{1}=M \sqrt{\frac{K}{1-M}}$, where $M=\frac{V_{o}}{V_{g}}$ and $K=\frac{2 L}{R_{L} T_{s}}=\frac{2 L I_{\mathrm{LED}}}{V_{o} T_{s}}[39]$.

\section{$\infty_{\infty}^{\infty}$}

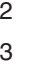
(a) 


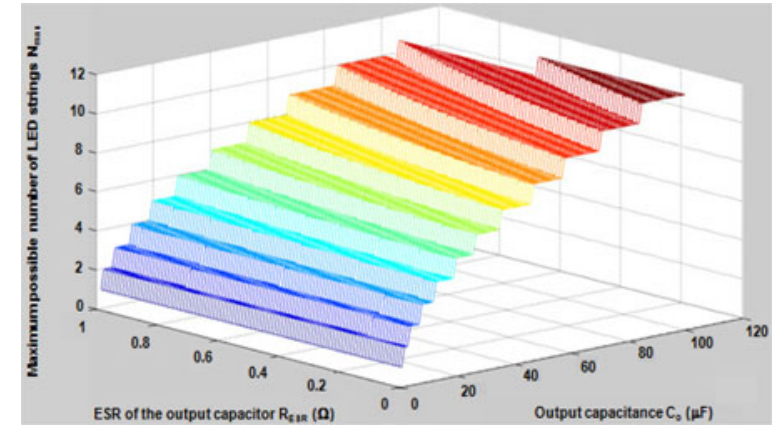

Fig. 10. Theoretical maximum achievable number of LED strings $\left(N_{\max }\right)$ versus the output capacitance $\left(C_{o}\right)$ and the capacitor ESR $\left(R_{\mathrm{ESR}}\right)$ for the scalable BCM-based SIMO scheme.

it is always recommended to choose an output capacitor with a smaller ESR, whenever possible. Fig. 10 shows the theoretical maximum total number of LED strings versus the output capacitance and capacitor ESR for the scalable BCM-based SIMO scheme, given an LED current of $80 \mathrm{~mA}$ and a maximum ripple current requirement of $40 \%$ P-P.

Intuitively, for a particular LED current, an increasing number of outputs can be achieved by using a larger output capacitor with the same ESR value. For instance, if the output capacitance is increased from 4.7 to $22 \mu \mathrm{F}$ (the ESR remains at $100 \mathrm{~m} \Omega$ ), the theoretical model based on (22) suggests that the maximum total number of LED strings can be increased from three to six. It is also interesting to note that the maximum number of outputs in SIMO has a stronger dependence on the output capacitance than the capacitor ESR, as shown in Fig. 10.

On the other hand, it is useful to study how the LED current affects the maximum achievable number of outputs in SIMO. As an example, assuming balanced load and two LEDs connected in series per string, a scalable BCM-based SIMO scheme is investigated with these parameter values: $L=47 \mu \mathrm{H}, C_{o}=$ $4.7 \mu \mathrm{F}, R_{\mathrm{ESR}}=100 \mathrm{~m} \Omega, V_{g}=15 \mathrm{~V}$, and $V_{o}=6.4 \mathrm{~V}$. The relationship between $N_{\max }$ and $I_{\mathrm{LED}}$ can be obtained by using (22) for different values of output voltage ripple $\Delta v_{o \_ \text {max }}$. Based upon the $I-V$ curve and/or SPICE model of the particular LED used, the corresponding output voltage ripple $\Delta v_{o \_ \text {max }}$ can be determined from the LED current ripple requirement $\Delta i_{\mathrm{LED}}$. The proposed design uses white LED [40] which is the target for LCD backlighting applications. For instance, a 20\%p-p current ripple corresponds to around $2 \%$ P-P voltage ripple and a $40 \%$ P-P current ripple corresponds to around 4\% -P voltage ripple. Fig. 11 shows a plot of $N_{\max }$ versus $I_{\mathrm{LED}}$ for $\Delta i_{\mathrm{LED}}$ ranging from 5\%p-p to $40 \%$ p-P. This plot is beneficial to a practical SIMO design in two ways. First, for a given LED current and current ripple requirement, the theoretical maximum number of LED strings viable under the scalable BCM-based SIMO scheme can be extracted directly from the plot. Second, the

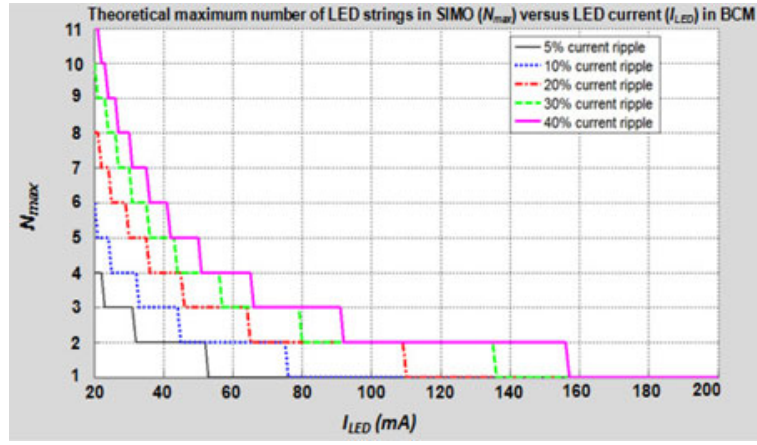

Fig. 11. Plot of theoretical maximum number of LED strings in SIMO $\left(N_{\max }\right)$ versus the LED current $\left(I_{\mathrm{LED}}\right)$ in the scalable BCM-based SIMO scheme.

maximum LED current allowed in order for a SIMO to remain 461 at the same scaling level can also be obtained from the plot. For 462 instance, given a $20 \%$ current ripple requirement (i.e., $\Delta i_{\mathrm{LED}}=463$ $20 \%$ p-P), a SIDO (dual-string) configuration is possible as long 464 as the LED current in each string is no more than $110 \mathrm{~mA}$. In 465 the event that an application demands an LED current greater 466 than $110 \mathrm{~mA}$, two options can be considered: 1) Relax the cur- 467 rent ripple requirement whenever possible. A wider tolerance 468 in $\Delta i_{\mathrm{LED}}$ is generally acceptable since the ripple frequency is 469 too high for the human eye to detect. 2) Operate the SIMO buck 470 LED driver in PCCM [17], [35], [36]. In PCCM, the floor of 471 the inductor current is raised by a nonzero dc offset $I_{\mathrm{DC}}$ which 472 distinguishes it from DCM. The proposed theoretical model can 473 be extended to PCCM by adding a dc component to the aver- 474 age inductor current. By going through similar calculations as 475 in DCM, the theoretical maximum number of outputs in SIMO 476 operating in PCCM is given by (24), as shown at the bottom of 477 the page. It is interesting to note that (24) continues to apply to 478 the cases of DCM and BCM. For instance, in DCM, $I_{\mathrm{DC}}=0 \quad 479$ and (24), therefore, reduces to (23).

In the event of unbalanced load with unequal current among the LED strings, the scalable DCM- or BCM-based SIMO scheme continues to hold. The only change is to replace $I_{\mathrm{LED}}$ in (22) and (23) by $\max \left(I_{\mathrm{LED}}\right)$, where $\max \left(I_{\mathrm{LED}}\right)$ denotes the largest LED current among all the LED strings. In other words, the maximum number of LED strings that can be realized in a SIMO buck LED driver is constrained by the largest LED current. Generally speaking, the input voltage $V_{g}$, output voltage $V_{o}$, and the current ripple requirement are typically fixed parameters defined in the design specification. Without making any hardware changes (i.e., $L$ and $C_{o}$ values are fixed), the primary design variable in (22) and (23) is the LED current $I_{\mathrm{LED}}$. In fact, the LED current is the dominant factor for determining the maximum possible number of outputs under the scalable $D C M-/ B C M$-based SIMO scheme. By knowing the maximum LED current required for a particular application, the theoretical

\section{1} 62 463 464 465 66 67 68 469

470

1

42

43

44

45

76

47
48

$$
N_{\text {max } \_ \text {PCCM }}=\text { floor }\left(\frac{1}{2 I_{\mathrm{LED}}} \times\left[\left(I_{\mathrm{DC}}+\left(1-D_{3}\right) I_{\mathrm{LED}}\right] \times\left[1+\sqrt{\left.1+\frac{2 C_{o}\left(\Delta v_{o} \max \right.}{\left.L\left[I_{\mathrm{LED}} R_{\mathrm{ESR}}\right)\left(V_{g}-V_{o}\right) D_{1}\left(1-D_{3}\right) I_{\mathrm{LED}}\right]^{2}}\right)}\right) .\right.\right.
$$


TABLE I

DESIGN SPECIFICATION OF A SISO BUCK LED DRIVER IN DCM

\begin{tabular}{|c|c|c|}
\hline Design Parameter & Value & Unit \\
\hline Input Voltage $\left(V_{g}\right)$ & 15 & $\mathrm{~V}$ \\
\hline Output Voltage $\left(V_{o}\right)$ & 6.32 & $\mathrm{~V}$ \\
\hline LED Forward Current $\left(I_{L E D}\right)$ & 80 & $\mathrm{~mA}$ \\
\hline Switching Frequency $\left(f_{s}\right)$ & 100 & $\mathrm{kHz}$ \\
\hline Inductor $(L)$ & 47 & $\mu \mathrm{H}$ \\
\hline Output Capacitor $\left(C_{o}\right)$ & 4.7 & $\mu F$ \\
\hline ESR of Output Capacitor $\left(R_{E S R}\right)$ & 100 & $\mathrm{~m} \Omega$ \\
\hline $\begin{array}{c}\text { Maximum LED Current Ripple } \\
\left(\Delta i_{L E D}\right)\end{array}$ & 40 & $\%_{\mathrm{P}-\mathrm{P}}$ \\
\hline $\begin{array}{c}\text { Maximum Output Voltage Ripple } \\
\left(\Delta v_{o}\right)\end{array}$ & 4 & $\%_{\mathrm{P}-\mathrm{P}}$ \\
\hline Duty Ratio of Idle Phase $\left(D_{3}\right)$ & $\geq 10$ & $\%$ \\
\hline
\end{tabular}

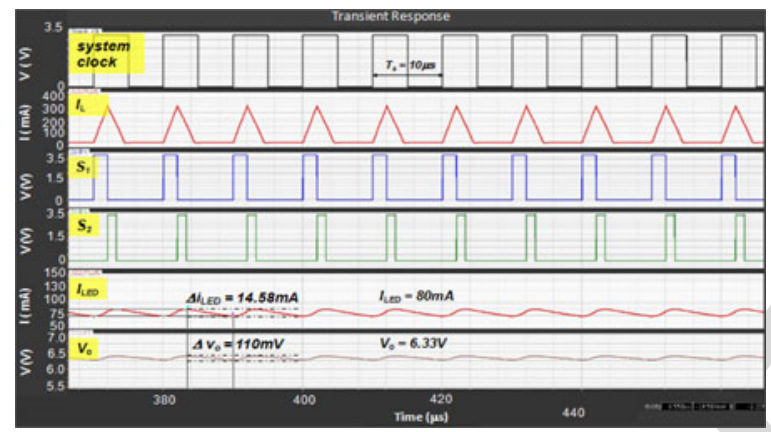

Fig. 12. Simulated steady-state waveforms for the SISO buck LED driver based on the design specification in Table I.

maximum achievable number of independently driven LED strings can be estimated in advance.

\section{Simulation Results}

Ideal macromodels based on the scalable DCM-based SIMO scheme were constructed and simulated in Cadence Spectre [27] in order to compare with the theoretical results in Section III. The design specification of a single-inductor single-output (SISO) buck converter is shown in Table I. The theoretical model based on (23) suggests that $N_{\text {max_DCM }}=1$, meaning only one LED string is viable. Fig. 12 shows the simulated steady-state waveforms of the inductor current $I_{L}$, the LED currents $I_{\mathrm{LED}}$, and the output voltages $V_{o}$ of a SISO buck LED driver. The simulated steady-state LED current $I_{\mathrm{LED}}$ is approximately $80 \mathrm{~mA}$ which meets the design target. The simulated LED current ripple $\Delta i_{\mathrm{LED}}$ is $18 \%$ P-P (also, the output voltage ripple $\Delta v_{o}$ is $1.7 \%$ p-p), which satisfies the maximum ripple requirement. Now, the SISO buck LED driver is transformed into SIDO by adding a second LED string. Fig. 13 shows the simulated steady-state waveforms from the resulting SIDO LED driver.

Despite the fact that the steady-state LED current in either string remains at $80 \mathrm{~mA}$, the LED current ripple is more than $40 \%$ p-p which violates the maximum ripple current requirement. Hence, the simulation results show that SIDO is not viable based on the design requirement which is consistent with the theoretical result. By increasing the switching frequency from

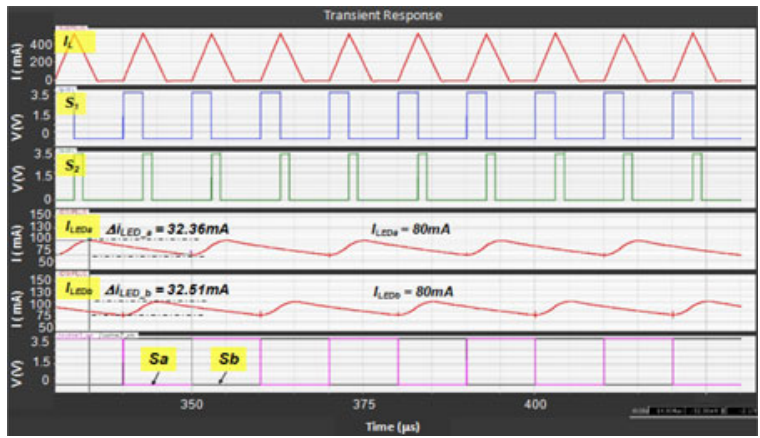

Fig. 13. Simulated steady-state waveforms showing SIDO is not viable since the $30 \%$ p-p maximum current ripple requirement is violated.

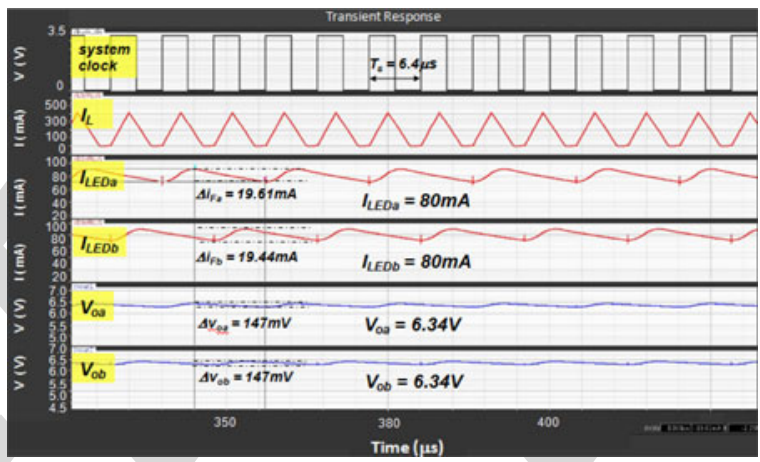

Fig. 14. Simulated steady-state waveforms showing SIDO is possible by increasing the switching frequency from 100 to $156.25 \mathrm{kHz}$

100 to $156.25 \mathrm{kHz}$ and keeping other parameters unchanged, 522 $N_{\text {max_DCM }}=2$ from (23). Fig. 14 shows the simulated wave- 523 forms for the corresponding signals in a SIDO buck LED driver. 524 The simulated LED current ripple $\Delta i_{\mathrm{LED}}$ is $24 \%$ P-P and the cor- 525 responding output voltage ripple $\Delta v_{o}$ is $2.3 \%$ P-P , both of which 526 satisfy their corresponding maximum ripple requirement. Con- 527 sequently, both the theoretical and simulation results show that 528 by increasing the switching frequency, a SIDO buck LED driver 529 in DCM is feasible. 530

A third LED string is added to the SIDO buck LED driver to 531 transform it into SIMO consisting of three independently driven 532 LED strings. The LED current in each string remains unchanged 533 at $80 \mathrm{~mA}$ as in the SISO or SIDO case. According to Fig. 11, 534 the theoretical model suggests that for $I_{\mathrm{LED}}=80 \mathrm{~mA}$, a scal- 535 able BCM-based SIMO scheme with a maximum of three LED 536 strings is feasible under the $40 \%$ P-P current ripple constraint. 537 The switching period $T_{s}$ is chosen to be $6 \mu$ s using (13) which 538 corresponds to a switching frequency of $166.67 \mathrm{kHz}$. Fig. 15539 shows the simulated waveforms from the resulting SIMO buck 540 LED driver. The simulated LED current ripple $\Delta i_{\mathrm{LED}}$ is around 541 $35 \%$ p-P and the output voltage ripple $\Delta v_{o}$ is $3.5 \%$ P-P, both of 542 which satisfy their respective maximum ripple constraint. As a 543 sanity check, the theoretical model based on (22) indeed sug- 544 gests that a maximum possible number of three independently 545 driven LED strings can be achieved in the scalable BCM-based 546 SIMO scheme. Hence, it is shown that the simulation result 547 agrees with the corresponding theoretical result. On the other 548 hand, it is important to examine the transient performance of the 549 


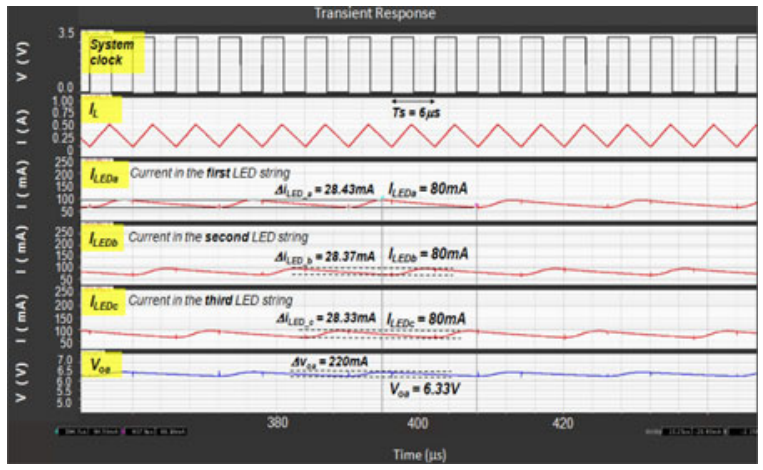

Fig. 15. Simulated steady-state waveforms of a three-string SIMO buck LED driver operating in $\mathrm{BCM}$

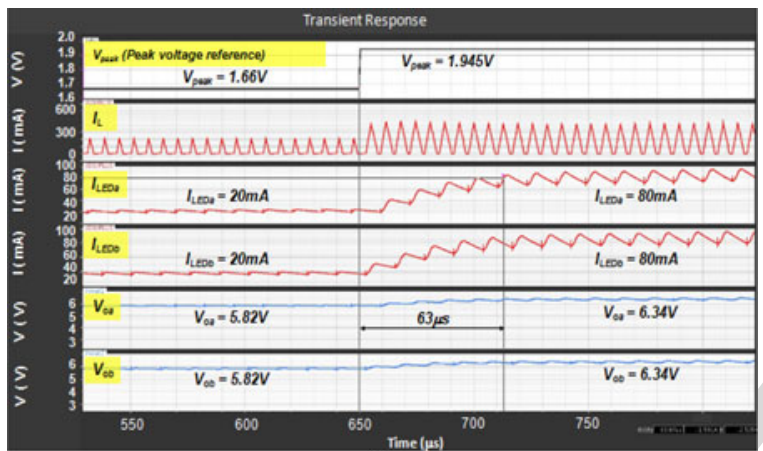

(a)

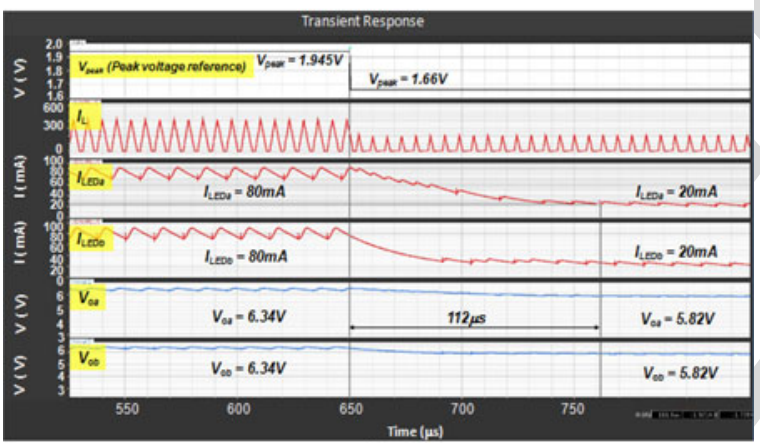

(b)

Fig. 16. Simulated transient waveforms for (a) peak reference step-up and (b) peak reference step-down response. proposed SIDO buck LED driver. The LED current is changed by adjusting the peak limit of the inductor current. By stepping up the peak voltage reference ( $V_{\text {peak }}$ in Fig. 1) from 1.660 to $1.945 \mathrm{~V}$ in $400 \mathrm{~ns}$, the peak inductor current limit is increased by approximately $200 \mathrm{~mA}$, leading to an increase in the nominal LED current from 20 to $80 \mathrm{~mA}$. The reference voltages $\left(V_{\text {ref } a}, V_{\text {ref } b}\right)$ are also stepped up from 100 to $340 \mathrm{mV}$ in order to maintain the same load current between the two LED strings. Conversely, by stepping down $V_{\text {peak }}$ from 1.945 to $1.660 \mathrm{~V}$, the LED current is reduced from 80 to $20 \mathrm{~mA}$. Fig. 16 shows the simulated transient behavior for the peak voltage reference step-up and step-down response.

In the case of step-up reference response, the LED current in either string settles to the steady-state nominal value of $80 \mathrm{~mA}$ within $63 \mu$ s. The output voltage reaches its target steady-state

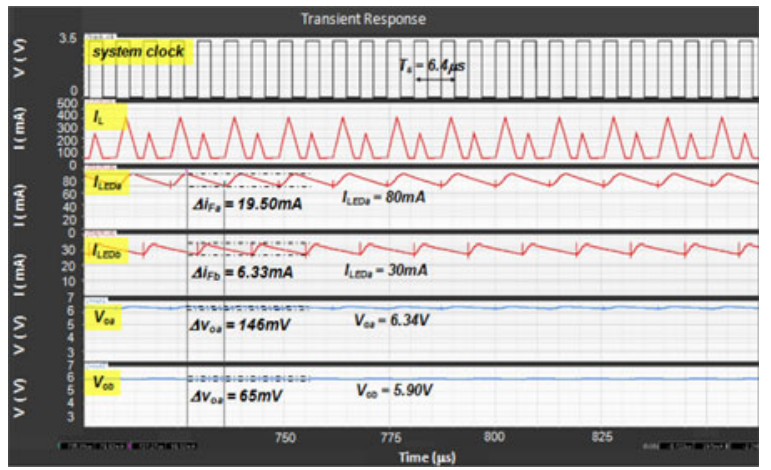

Fig. 17. Simulated steady-state waveforms of a SIDO buck LED driver with unbalanced load.

value of $6.34 \mathrm{~V}$. In the case of step-down reference response, the LED current in either string settles to the steady-state nominal value of $20 \mathrm{~mA}$ in less than $112 \mu \mathrm{s}$. The output voltage settles to its new steady-state value of $5.82 \mathrm{~V}$ without oscillations. Hence, the simulation results show that the closed-loop system remains in stable condition in response to a peak voltage reference transient.

The effectiveness of the proposed SIDO converter to drive unbalanced load is also investigated. As an example, the first and second LED strings require an average current value of 80 and $30 \mathrm{~mA}$, respectively. Unlike the balanced load case with a constant peak inductor current limit, two distinct peak current limits are employed for unbalanced load such that two different average inductor (or load) current values can be generated in alternate clock cycles. Fig. 17 depicts the simulated steady-state waveforms from the SIDO buck LED driver with unbalanced load. The simulation results show that the first and second LED strings are regulated with an average current value of 80 and $30 \mathrm{~mA}$, respectively. For the first string, the simulated current ripple is $24.38 \%$ P-P and the output voltage ripple is $2.3 \%$ P-P. Also, for the second string, the simulated current ripple is $21.1 \%$ p-p and the output voltage ripple is $1.1 \%$ p-P. Either string meets the maximum ripple requirements. The simulation results demonstrate that the proposed SIDO converter is capable of delivering unequal currents to the two LED strings simultaneously.

\section{EXPERIMENTAL RESULTS}

The proposed SIDO buck LED driver was implemented on a field-programmable gate array (FPGA)-based hardware prototype in accordance with the design specification provided in Table I. The switching frequency is increased to $156.25 \mathrm{kHz}$ in order to satisfy the LED current ripple requirement. A photo of the experimental setup is shown in Fig. 18. The power stage of the buck converter consists of discrete ICs from International Rectifier such as power MOSFETs (IRF7828), dual-channel gate driver (IR2110), and output switches (IRF9388), as well as surface-mount inductor and low-ESR capacitors. In actual implementation, the top level of the proposed digital controller is partitioned into two major functional blocks. The functionality of the first block is to control the switching action of the power 


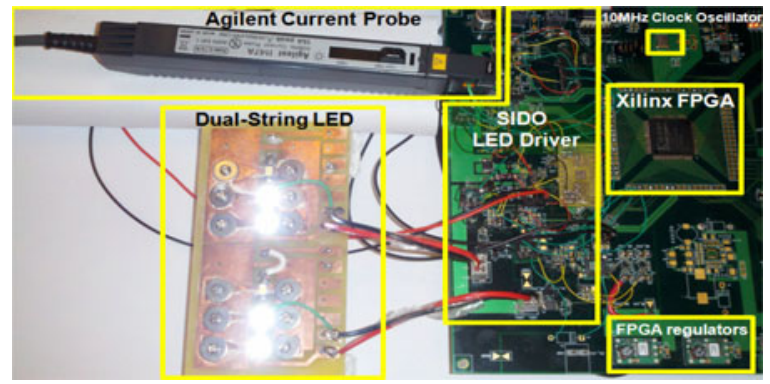

Fig. 18. Experimental setup for the proposed SIDO buck LED driver.

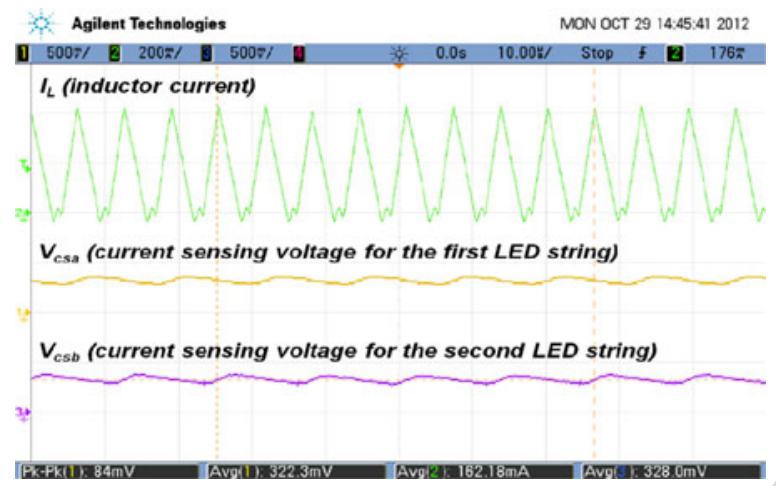

Fig. 19. Measured waveforms for inductor current and current sense feedback voltage.

stage by detecting the peak-crossing and zero-crossing events of the inductor current. It was implemented in hierarchical gatelevel schematics using primitives and macros available from the Xilinx Spartan-3 Generation library. Dead-time logic is included to prevent shoot-through current of the power switches. The second logical block is used to control the switching sequence of the two output switches by continuously monitoring the current-sense feedback signals. It was modeled as an FSM in Verilog RTL. Only one of the two output switches can be ON and the other must be OFF per switching cycle. Dead-time logic is also added to prevent cross conduction between outputs. The two logical blocks are synchronized by the system clock to ensure that the high-side power switch and the output switches are triggered from the same clock edge. The entire digital controller was implemented with Xilinx Spartan-3E (XC3S250E) FPGA. The quasi-hysteretic control logic was realized using 4-ns fast comparators (AD8611 from Analog Devices) and semicustom synchronous logic.

Using a current sensing resistor of $4 \Omega$ and reference voltage of $320 \mathrm{mV}$, the target current in each of the two LED strings is $80 \mathrm{~mA}$. Fig. 19 shows the current sensing feedback voltage $\left(V_{\mathrm{csa}}, V_{\mathrm{csb}}\right)$ from which the corresponding average load current can be obtained, i.e., $I_{\mathrm{LED}}=V_{c s} / R_{c s}$. The average inductor current is measured to be $162 \mathrm{~mA}$, which is the sum of the load currents in both LED strings. The average current values in the first and second LED string are measured to be around 80.6 and $82 \mathrm{~mA}$, respectively. The measured LED current ripple $\Delta i_{\mathrm{LED}}$ in either string is around $26 \%$ p-P, which is reasonably close to the simulated current ripple of $24 \%$ p-p. In addition, the nominal output voltages in the first and second string are

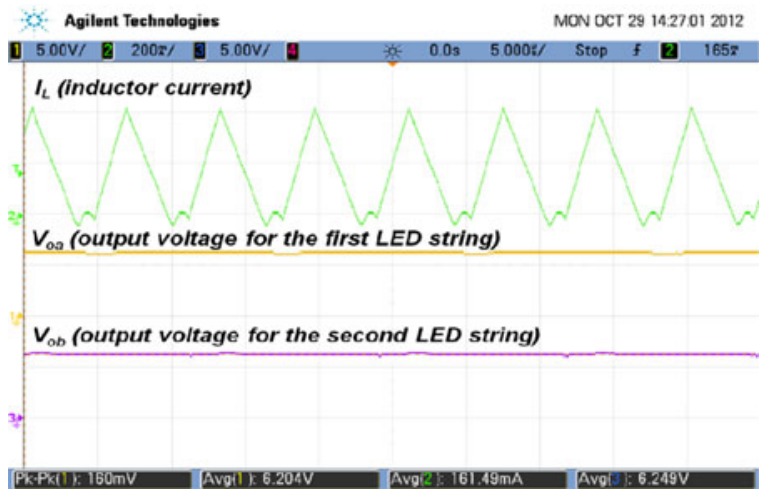

Fig. 20. Measured waveforms for inductor current and output voltage in either LED string.

measured to be 6.204 and $6.249 \mathrm{~V}$, respectively, as shown in Fig. 20. Under this balanced load condition, the measured current and voltage values are in close agreement between the two LED strings. The output voltage ripple is also measured to be around $2.57 \%$ P-P, compared to $2.3 \%$ P-P from simulation. Therefore, the experimental results are shown to be consistent with the corresponding simulation ones. On the other hand, the measured power conversion efficiency of the proposed SIDO converter is $80 \%$ which is comparable to conventional driving topologies [41]. The efficiency can be further increased by employing a current-sensing resistor with a smaller value.

The transient response of the proposed SIDO buck LED driver is verified experimentally by measuring its peak voltage reference step response. An 8-bit digital-to-analog converter (AD558 from Analog Devices) is used to enable programming of the peak voltage reference $V_{\text {peak }}$ and the current-sense voltage references $\left(V_{\text {ref } a}, V_{\text {ref } b}\right)$ by the Xilinx FPGA. The measured waveforms of the inductor current and the voltage at the current sensing nodes in response to a peak voltage reference step are shown in Fig. 21. The settling time of the transient response is also measured and compared with the simulated settling time. For the step-up response, it is observed that the current-sensing voltage $V_{\text {csa }}$ in the first LED string steps up from 81.8 to $325.4 \mathrm{mV}$, which corresponds to an increase in the average load current from 20.5 to $81.3 \mathrm{~mA}$. Similarly, the current-sensing voltage $V_{\text {csb }}$ in the second LED string steps up from 94.1 to $327.6 \mathrm{mV}$, which corresponds to an increase in the average load current from 23.5 to $81.9 \mathrm{~mA}$. The settling time for the step-up response is measured to be $67 \mu \mathrm{s}$, compared to $63 \mu$ s from simulation. The measured results for the step-down response are the reverse of those from the step-up response. The only difference is that it takes longer for the step-down transient to settle. The settling time for the step-down response is measured to be $115 \mu \mathrm{s}$, compared to $112 \mu \mathrm{s}$ from simulation. The measured settling times are shown to be very close to the simulated ones. The experimental results confirm that the system remains in stable condition when it is perturbed by the peak voltage reference transient.

The unbalanced load scenario in the proposed SIDO buck LED driver is also verified experimentally. The measured average load current values in the first and second LED string are 


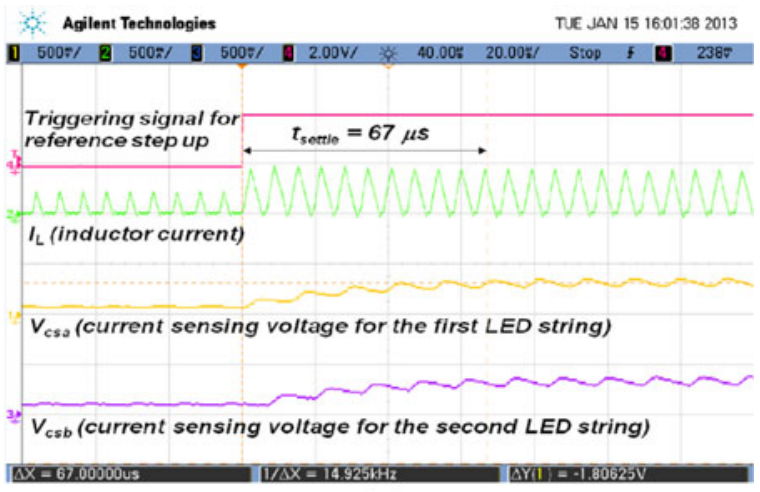

(a)

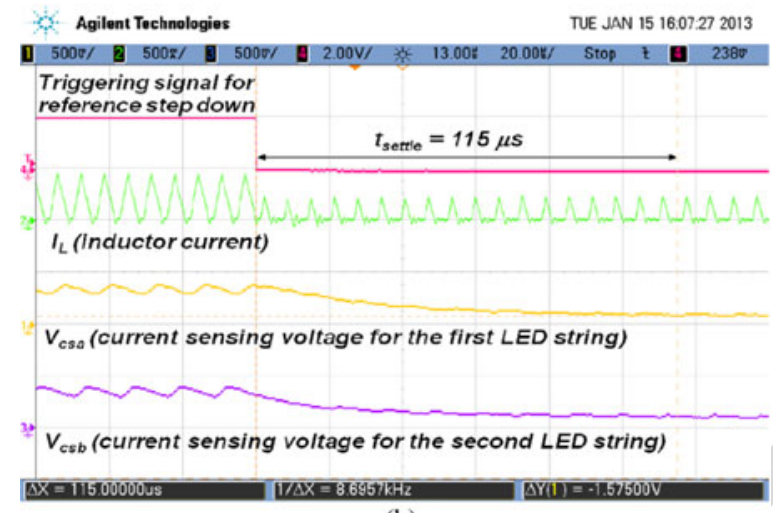

(b)

Fig. 21. Measured transient waveforms in response to (a) peak reference stepup and (b) peak reference step-down.

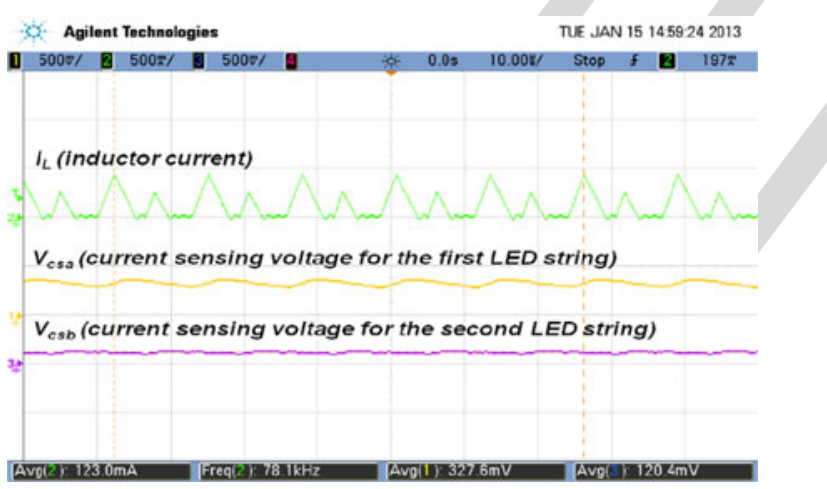

Fig. 22. Measured waveforms for inductor current and current sense voltages.

around 81.9 and $30.1 \mathrm{~mA}$, respectively. Fig. 22 shows the measured waveforms for the inductor current and the current-sensing voltage per string. The inductor current waveform indicates that the proposed driver operates in DCM with two distinct peak current limits. Fig. 23 shows the measured inductor current and the output voltage in either string. The measured output voltage values in the first and second LED string are 6.22 and $5.70 \mathrm{~V}$, respectively. The experimental results demonstrate that the proposed driver is capable of driving two independent LED strings concurrently with different load current.

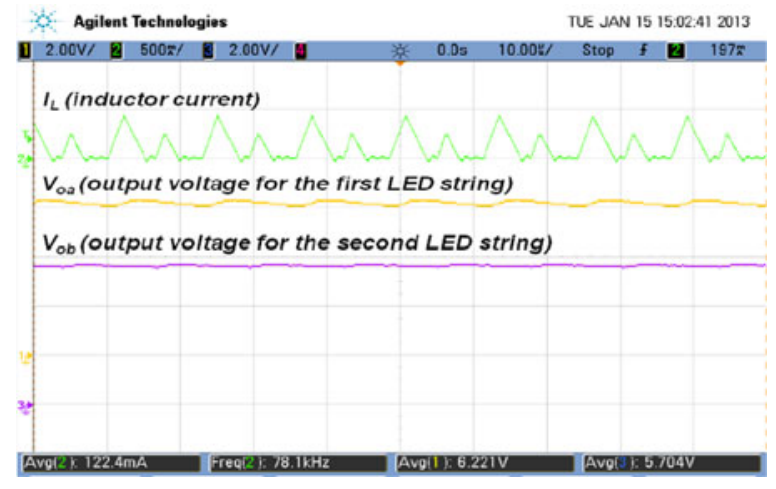

Fig. 23. Measured waveforms for inductor current and output voltages.

\section{CONCLUSION}

The proposed SIDO buck LED driver was implemented in 687 FPGA-based hardware. The experimental results correlate well 688 with simulation ones. The scalability of the proposed SIDO buck 689 LED driver to SIMO is closely examined. A general formula 690 for determining the theoretical maximum achievable number of 691 LED strings in SIMO is derived. The simulation results were 692 shown to be consistent with those obtained from the theoret- 693 ical model for the same design parameter values. The quasi- 694 hysteretic digital control scheme does not require loop com- 695 pensation which simplifies the control loop design and reduces 696 component count. In addition, the proposed SIMO architecture 697 offers the advantage of driving a larger number of parallel LED 698 strings without being limited by the maximum current rating of 699 the LED. It also enables dimming for the LED strings without 700 additional dimming transistors. Local bus voltage and current 701 optimization in each individual LED string compensates for the 702 variability of the LED's forward voltage, which reduces power 703 loss and enables mixing of white LEDs from different bins to 704 lower LED costs.

\section{REFERENCES}

[1] C.-Y Hsieh, C.-Y. Yang, and K.-H. Chen, "A charge-recycling buck-store and boost-restore (BSBR) technique with dual outputs for RGB LED backlight and flashlight module," IEEE Trans. Power Electron., vol. 24, no. 8, pp. 1914-1925, Aug. 2009.

[2] S.-Y. Lee, J.-W. Kwon, H.-S. Kim, M.-S. Choi, and K.-S. Byun, "New design and application of high efficiency LED driving system for RGBLED backlight in LCD display," in Proc. 37th IEEE Power Electron. Spec. Conf., 2006, pp. 1-5.

[3] H.-J. Chiu, Y.-K. Lo, J.-T. Chen, S.-J. Cheng, C.-Y. Lin, and S.-C. Mou, "A high-efficiency dimmable led driver for low-power lighting applications," IEEE Trans. Ind. Electron., vol. 57, no. 2, pp. 735-743, Feb. 2010.

[4] C.-H. Liu, C.-Y. Hsieh, Y.-C. Hsieh, T.-J. Tai, and K.-H. Chen, "SAR Controlled adaptive off-time technique without sensing resistor for achieving high efficiency and accuracy led lighting system," IEEE Trans. Circuits Syst. I, Reg. Papers, vol. 57, no. 6, pp. 1384-1394, Jun. 2010.

[5] C.-H. Wu and C.-L. Chen, "High-efficiency current-regulated charge pump for a white LED driver," IEEE Trans. Circuits Syst. II, Exp. Briefs, vol. 56 no. 10, pp. 614-618, Oct. 2009.

D. Belloni, F. Gozzini, C. Bazzani, and A. Baschirotto, "A 725 $0.18 \mu \mathrm{m}$ CMOS 91\%-efficiency 0.1-to-2 A scalable buck-boost DC-DC 726 converter for LED drivers," in Proc. ISSCC Dig. Tech. Papers, Feb. 2012， 727 pp. 280-281.

[7] S. Rao, Q. Khan, S. Bang, D. Swank, A. Rao, W. Mclntyre, and 729 P. K. Hanumolu, “A 1.2 A buck-boost LED driver with $13 \%$ efficiency 730 
improvement using error-averaged SenseFET-Based current sensing," in Proc. ISSCC Dig. Tech. Papers, Feb. 2011, pp. 238-239.

[8] S.-I. Hong, J.-W Han, D.-H. Kim, and O.-K. Kwon, "A double-loop control LED backlight driver IC for medium-sized LEDs," in Proc. ISSCC Dig. Tech. Papers, Feb. 2010, pp. 116-117.

[9] M.-H. Huang and K.-H. Chen, "Single-inductor multi-output (SIMO) DC-DC converters with high light-load efficiency and minimized crossregulation for portable devices," IEEE J. Solid-State Circuits, vol. 44, no. 4, pp. 1099-1111, Apr. 2009.

10] E. Bonizzoni, F. Borghetti, P. Malcovati, F. Maloberti, and B. Niessen, "A $200 \mathrm{~mA} 93 \%$ peak efficiency single-inductor dual-output DC-DC buck converter," in Proc. IEEE ISSCC Dig. Tech. Papers, Feb. 2007, pp. 526619.

11] C.-Y. Hsieh and K.-H. Chen, "Boost DC-DC converter with fast reference tracking (FRT) and charge-recycling (CR) techniques for high-efficiency and low-cost LED driver," IEEE J. Solid-State Circuits, vol. 44, no. 9, pp. 2568-2580, Sep. 2009.

[12] H. Chen, Y. Zhang, and D. Ma, "A SIMO parallel-string driver IC for dimmable LED backlighting with local bus voltage optimization and single time-shared regulation loop," IEEE Trans. Power Electron., vol. 27, no. 1, pp. 452-462, Jan. 2012.

13] D. Ma, W.-H. Ki, P. K. T. Mok, and C.-Y. Tsui, "Single-inductor multipleoutput switching converters with bipolar outputs," in Proc. IEEE Int. Symp. Circuits Syst., May 2001, vol. 3, pp. 301-304.

14] D. Ma, W.-H. Ki, C.-Y. Tsui, and P. K. T. Mok, "A 1.8 V single-inductor dual-output switching converter for power reduction techniques," in Proc. IEEE Symp. VLSI Circuits, Jun. 2001, pp. 137-140.

[15] W.-H. Ki and D. Ma, "Single-inductor multiple-output switching converters," in Proc. 32nd IEEE Power Electron. Spec. Conf., Jun. 2001, vol. 1, pp. 226-231.

16] D. Ma, W.-H. Ki, C.-Y. Tsui, and P. K. T. Mok, "Single-inductor multipleoutput switching converters with time-multiplexing control in discontinuous conduction mode," IEEE J. Solid-State Circuits, vol. 38, no. 1, pp. 89-100, Jan. 2003.

17] D. Ma, W.-H. Ki, and C.-Y. Tsui, "A pseudo-CCM/DCM SIMO switching converter with freewheel switching," IEEE J. Solid-State Circuits, vol. 38, no. 6, pp. 1007-1014, Jun. 2003.

[18] D. Kwon and G. A. Rincón-Mora, "Single-inductor multiple-output switching DC-DC converters," IEEE Trans. Circuits Syst. II, Exp. Briefs, vol. 56, no. 8, pp. 614-618, Aug. 2009.

[19] K.-S. Seol, Y.-J. Woo, G.-H. Cho, G.-H. Gho, and J.-W. Lee, “A synchronous multioutput step-up/down DC-DC converter with return current control," IEEE Trans. Circuits Syst. II, Exp. Briefs, vol. 56, no. 3, pp. 210214, Mar. 2009

[20] C.-W. Leng, C.-H. Yang, and C.-H. Tsai, "Digital PWM controller for SIDO switching converter with time-multiplexing scheme," in Proc. Int. Symp. VLSI Design Autom. Test, Apr. 2009, pp. 52-55.

[21] Y.-J. Moon, Y.-S. Roh, J.-C. Gong, and C. Yoo, "Load-independent current control technique of a single-inductor multiple-output switching DC-DC converter," IEEE Trans. Circuits Syst. II, Exp. Briefs, vol. 59, no. 1, pp. 5054, Jan. 2012.

[22] F. Su, W.-H. Ki, and C.-Y. Tsui, "Ultra fast fixed-frequency hysteretic buck converter with maximum charging current control and adaptive for DVS applications," IEEE J. Solid- State Circuits, vol. 43, no. 4, pp. 815-822, Apr. 2008.

[23] W.-H. Ki, K.-M. Lai, and C. Zhan, "Charge balance analysis and state transition analysis of hysteretic voltage mode switching converters," IEEE Trans. Circuits Syst. I, Reg. Papers, vol. 58, no. 5, pp. 1142-1153, May 2011.

24] K. K.-S. Leung and H. S.-H. Chung, "Dynamic hysteresis band control of the buck converter with fast transient response," IEEE Trans. Circuits Syst. II, Exp. Briefs, vol. 52, no. 7, pp. 398-402, Jul. 2005.

25] H. Eachempatti, S. Ganta, J. Silva-Martinez, and H. Martínez-Garcia, "SIDO buck converter with independent outputs," in Proc. 53rd IEEE Int. Midwest Symp. Circuits Syst., Aug. 2010, pp. 37-40.

[26] F. Su and W.-H. Ki, "Digitally assisted quasi-V" ${ }^{2}$ hysteretic buck converter with fixed frequency and without using Large-ESR capacitor," in Proc. ISSCC Dig. Tech. Papers, Feb. 2009, pp. 446-447.

[27] Cadence Spectre Circuit Simulator Datasheet [Online]. Available: http://www.cadence.com/products/cic/spectre_circuit/pages/default.aspx

[28] M. Doshi and R. Zane, "Digital architecture for driving large LED arrays with dynamic bus voltage regulation and phase shifted PWM," in Proc. Appl. Power Electron. Conf., Feb. 2007, pp. 287-293.

29] W.-S. Oh, D. Cho, K.-M. Cho, G.-W. Moon, B. Yang, and T. Jang, "A novel two-dimensional adaptive dimming technique of $\mathrm{X}-\mathrm{Y}$ channel drivers for
LED backlight system in LCD TVs," J. Display Technol., vol. 5, no. 1, 806 pp. 20-26, Jan. 2009.

[30] Datasheet: LP8545, "LP8545 High-efficiency LED backlight driver for notebooks," Texas Instruments Incorporated, [Online] Available: 809 http://www.ti.com/general/docs/lit/getliterature.tsp?genericPartNumber $=810$ lp8545\&fileType $=$ pdf

[31] Datasheet: MAX16814, "Integrated, 4-channel, high-brightness LED 812 driver with high-voltage DC-DC controller," Maxim Integrated Prod- 813 ucts, Inc., [Online], Available: http://datasheets.maximintegrated.com/ 814 en/ds/MAX16814.pdf

[32] K. H. Loo, W. K. Lun, S. C. Tan, Y. M. Lai, and C. K. Tse, "On driving techniques for LEDs: Toward a generalized methodology," IEEE Trans. Power Electron., vol. 24, no. 12, pp. 2967-2976, Dec. 2009.

[33] W. K. Lun, K. H. Loo, S. C. Tan, Y. M. Lai, and C. K. Tse, "Bilevel current driving technique for LEDs," IEEE Trans. Power Electron., vol. 24, no. 12, pp. 2920-2932, Dec. 2009

[34] S. C. Tan, "General n-level driving approach for improving electrical-tooptical energy-conversion efficiency of fast-response saturable lighting devices," IEEE Trans. Ind. Electron., vol. 57, no. 4, pp. 1342-1353, Apr. 2010.

[35] D. Ma and W.-H. Ki, "Fast transient PCCM switching converter with freewheel switching control," IEEE Trans. Circuits Syst. II, Exp. Briefs, vol. 54, no. 9, pp. 825-829, Sep. 2007.

[36] D. Ma, W.-H. Ki, and C.-Y. Tsui, "Single-inductor multiple-output switching converters in PCCM with freewheel switching," U.S. Patent 7432614 Oct. 7, 2008.

[37] Application Note: AN-1656, "Design challenges of switching LED drivers," Texas Instruments Incorporated, [Online] Available: http:// www.ti.com/general/docs/lit/getliterature.tsp?literatureNumber $=$ snva253 $\&$ fileType $=$ pdf

[38] Solutions Guide, "LED drivers for high-brightness lighting," Texas Instruments Incorporated, [Online], Available: http://www.ti.com/ general/docs/lit/getliterature.tsp?baseLiteratureNumber $=$ snvy001

[39] R. W. Erickson and D. Maksimovic, Fundamentals of Power Electronics, 2nd ed. New York, NY, USA: Springer, 2001.

[40] Datasheet: SMD5730 "SMD 5730 White LED Datasheet" APT Electronics Ltd., [Online], Available: http://www.apt-hk.com/en/product/?95.html

[41] Z. Ye, F. Greenfeld, and Z. Liang, "Design considerations of a high power factor SEPIC converter for high brightness white LED lighting applications," in Proc. IEEE Power Electron. Spec. Conf., 2008, pp. 2657-2663.
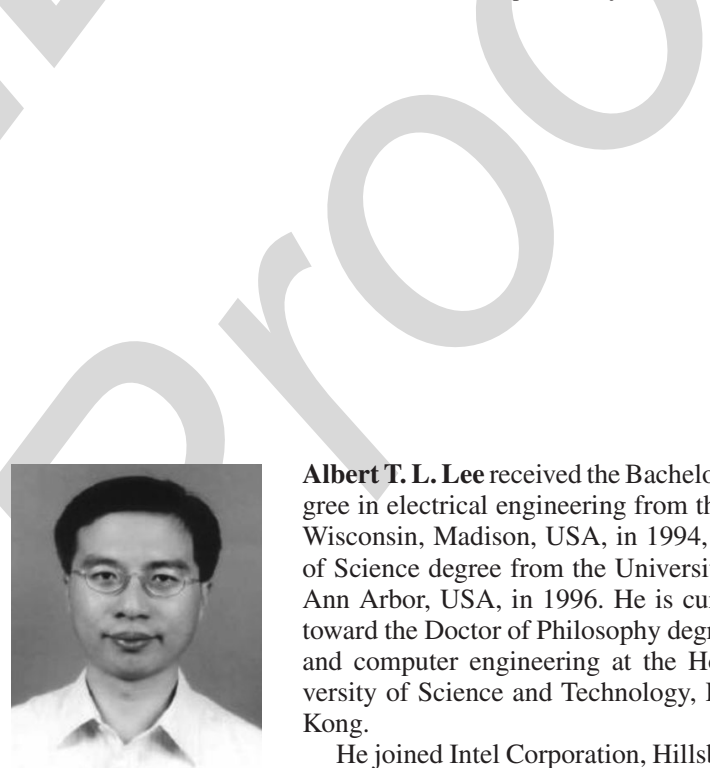

Albert T. L. Lee received the Bachelor of Science degree in electrical engineering from the University of Wisconsin, Madison, USA, in 1994, and the Master of Science degree from the University of Michigan, Ann Arbor, USA, in 1996. He is currently working toward the Doctor of Philosophy degree in electronic and computer engineering at the Hong Kong University of Science and Technology, Kowloon, Hong Kong.

He joined Intel Corporation, Hillsboro, OR, USA, in 1996 as a Senior Component Design Engineer and was involved in the development of Intel's P6 family microprocessors. In 2001, he served as a Senior Corporate Application Engineer in the System-Level Design Group at Synopsys Inc., Mountain View, CA, USA. In 2003, he joined the Hong Kong Applied Science and Technology Research Institute Company Ltd. and served as EDA Manager in the Wireline Communications Group. In 2006, he joined the Giant Electronics Limited as Hardware Design Manager and became Associate General Manager in 2008. His research interests include mixed-signal system-level design, LED driver, power management system, and very large scale integration circuits. 


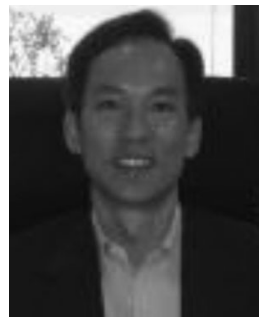

Johnny K. O. Sin (S'79-M'88-SM'96-F'12) received the B.A.Sc., M.A.Sc., and Ph.D. degrees in electrical engineering from the University of Toronto, Toronto, ON, Canada, in 1981, 1983, and 1988, respectively.

From 1988 to 1991, he was a Senior Member of the research staff of Philips Laboratories, Briarcliff Manor, NY, USA. In August 1991, he joined the Department of Electronic and Computer Engineering, The Hong Kong University of Science and Technology, Kowloon, Hong Kong, where he has been a Full Professor since 2001. He is the holder of 13 patents, and the author of more than 270 papers in technical journals and refereed conference proceedings. His research interests include microelectronic and nanoelectronic devices and fabrication technology, particularly novel power semiconductor devices and ICs, and system-on-a-chip applications using CMOS and power transistors and siliconembedded magnetic and capacitive devices.

Dr. Sin was an Editor for the IEEE ELECTRON DEVICES LETTERS from 1998 to 2010. He is a member of the Power Devices and IC's Technical Committee of the IEEE Electron Devices Society. He is also a Technical Committee member of the International Symposium on Power Semiconductor Devices and IC's. He is a Fellow of the IEEE for contributions to the design and commercialization of power semiconductor devices.

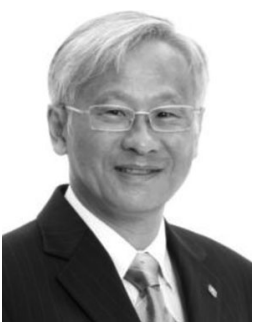

Philip C. H. Chan (SM'97-F'07) received the Bachelor of Science degree in electrical engineering from the University of California at Davis, Davis, USA, in 1973, and the Master of Science and Doctor of Philosophy degrees in electrical engineering from the University of Illinois at Urbana-Champaign, Urbana, USA, in 1975 and 1978, respectively.

He later joined Intel Corporation, Santa Clara, CA, USA, in 1981 and became a Senior Project Manager in Technology Development. He joined the Hong Kong University of Science and Technology (HKUST) in 1991 as a founding member. He served at HKUST as the Associate Dean of Engineering and Head of the Department of Electronic and Computer Engineering. He became the Dean of Engineering in September 2003. He joined the Hong Kong Polytechnic University, Hong Kong, in 2010 as the Deputy President and Provost. His research interests include very large scale integration devices, circuits, electronic packaging, and integrated sensors.

Dr. Chan received the ECE Distinguished Alumni Award from the University of Illinois, Urbana-Champaign in 2010. 
912 Q1. Author: Please provide year information in Refs. [27], [30], [31], [37], [38], and [40]. 


\title{
Scalability of Quasi-Hysteretic FSM-Based Digitally Controlled Single-Inductor Dual-String Buck LED Driver to Multiple Strings
}

\author{
Albert T. L. Lee, Johnny K. O. Sin, Fellow, IEEE, and Philip C. H. Chan, Fellow, IEEE
}

\begin{abstract}
There has been growing interest in single-inductor multiple-output (SIMO) dc-dc converters due to its reduced cost and smaller form factor in comparison with using multiple singleoutput converters. An application for such a SIMO-based switching converter is to drive multiple LED strings in a multichannel LED display. This paper proposes a quasi-hysteretic finite-statemachine-based digitally controlled single-inductor dual-output buck switching LED driver operating in discontinuous conduction mode (DCM) and extends it to drive multiple outputs. Based on the time-multiplexing control scheme in DCM, a theoretical upper limit of the total number of outputs in a SIMO buck switching LED driver for various backlight LED current values can be derived analytically. The advantages of the proposed SIMO LED driver include reducing the controller design complexity by eliminating loop compensation, driving more LED strings without limited by the maximum LED current rating, performing digital dimming with no additional switches required, and optimization of local bus voltage to compensate for variability of LED forward voltage $V_{F}$ in each individual LED string with smaller power loss. Loosely binned LEDs with larger $V_{F}$ variation can, therefore, be used for reduced LED costs.
\end{abstract}

Index Terms-Boundary conduction mode (BCM), discontinuous conduction mode (DCM), finite-state machine (FSM), singleinductor dual-output (SIDO), single-inductor multiple-output (SIMO).

\begin{abstract}
A N LED driver is essentially a current source (or sink) which maintains a constant current required for achieving the desired color and luminous flux from an array of LEDs. A number of highly efficient switching LED drivers have been reported in the literature and their primary objective is to achieve high power conversion efficiency [1]-[11]. Besides efficiency, another important consideration is the scalability of the existing single-inductor dual-output (SIDO) switching converter to drive multiple independent LED strings in a single-inductor multiple-output (SIMO) topology for reduced cost and smaller
\end{abstract}

Manuscript received November 7, 2012; revised January 22, 2013; accepted March 11, 2013. Recommended for publication by Associate Prof. J. M. Alonso.

A. T. L. Lee and J. K. O. Sin are with the Department of Electronic and Computer Engineering, The Hong Kong University of Science and Technology, Kowloon, Hong Kong (e-mail: alee@ust.hk; eesin@ust.hk).

P. C. H. Chan is with Hong Kong Polytechnic University, Hong Kong (e-mail: Philipch.Chan@inet.polyu.edu.hk).

Color versions of one or more of the figures in this paper are available online at http://ieeexplore.ieee.org.

Digital Object Identifier 10.1109/TPEL.2013.2253804 form factor [12]. However, in practice, only a finite number of outputs can be served by each LED driver.

The prior arts of SIMO switching converter use either one of two ways to distribute energy from a single power supply to multiple outputs with a single inductor, namely multiple energizing phases [13]-[20] and single energizing phase per switching cycle [21]. The former with time-multiplexing control leads to much better suppression of cross regulation because the outputs are decoupled in time. In this paper, a quasi-hysteretic finite-state machine (FSM)-based digital control scheme is employed in a SIDO buck LED driver consisting of two independent parallel strings operating in discontinuous conduction mode (DCM). The extension of this SIDO architecture to SIMO is formally investigated. The proposed SIMO-based switching buck LED driver enables separate control of the three primary colors (red, green, and blue), thereby offering more flexibility for color mixing. The rest of this paper is organized as follows. Section II introduces the proposed quasi-hysteretic FSM-based digital controller for a SIDO switching buck LED driver operating in DCM. Section III provides a theoretical analysis on the scalability of the proposed digital control scheme from SIDO to SIMO and suggests a general formula for determining the theoretical upper bound in the total number of outputs in SIMO. Section IV shows Cadence Spectre simulation results that are used to verify the theoretical model. Section V contains the experimental results for the proposed digitally controlled SIDO buck LED driver. Section VI concludes our research effort.

\section{QUASI-HySTERETIC FSM-BASED DigITAL CONTROL} FOR SIDO LED DRIVER

A SIDO switching converter with time-multiplexing control scheme operating in DCM was first reported in [13]-[16]. With such kind of time-multiplexing control scheme, a SIDO converter can easily be extended to drive multiple outputs and it exhibits negligible cross regulation in DCM. A SIMO parallelstring LED driver operating in DCM has recently been reported [12]. It uses an analog-based controller with dominant pole compensation for stability, and time-multiplexing control in DCM is employed to suppress cross regulation among the LED strings. Unlike conventional pulse width modulation (PWM)based analog controllers, the proposed digital controller utilizing quasi-hysteretic control does not require any compensation circuits because of its inherent stability [22]-[24], hence simplifying the control loop design and reducing the component count and cost. Quasi-hysteretic control offers a good 


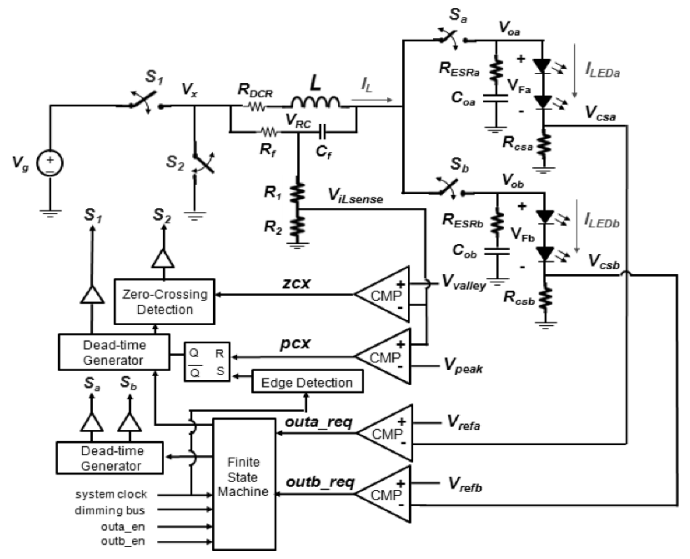

Fig. 1. System architecture of the quasi-hysteretic FSM-based digitally controlled SIDO buck LED driver.

compromise between traditional fixed-frequency PWM control and pure hysteretic control with variable switching frequency. In the proposed design, an external clock is used to synchronize the buck LED driver which switches at a fixed frequency. Fast comparators are used to control the on-time of the highside and low-side power switches by monitoring the inductor current. This is particularly suitable for lighting applications where variable switching frequencies are not desirable. The reconfigurability and scalability of a digital controller makes it especially attractive for SIMO.

A major drawback of the previously proposed SIMO LED driver operating in DCM [12] is that since the LED current is discontinuous, the LED endures a higher maximum peak current for the same average current required. In other words, the LED could potentially be operating close to its absolute maximum current rating, thereby increasing the current stress and possibly shortening the operating lifetime of the LED. In their approach, the LED current scales with the number of LED strings in SIMO. Hence, the maximum current rating of the LED unnecessarily restricts the maximum achievable number of LED strings which can be implemented in SIMO. In the proposed design, the LED current is always continuous and the LED can be regulated very close to the target average current value which is much lower than its maximum current rating. During the time interval when the output switch is OFF, the output capacitor, acting as a constant current source, continues to discharge its current to the corresponding LED string. When the output switch is ON, the power stage is reconnected to the LED string and the inductor current is transferred to the output capacitor and the LED string simultaneously. The current-sense feedback control ensures that the LED current is maintained at the desired dc level. Hence, a time-continuous current is supplied to the LED string. Consequently, the LED current does not scale with the number of LED strings in the proposed SIMO architecture, making it possible to drive more LED strings without inducing too much stress on the LEDs. Fig. 1 shows the system architecture of the proposed quasi-hysteretic FSM-based digitally controlled SIDO buck switching LED driver which takes into account the parasitic effects including the dc resistance (DCR) of the inductor $L$ and equivalent series resistance (ESR) of the output capacitors $\left(C_{o a}, C_{o b}\right)$. The two independently driven LED strings share the same inductor $L$ and the two main power switches $\left(S_{1}, S_{2}\right)$ of the buck converter. The output switches $\left(S_{a}, S_{b}\right)$ enable the charge stored in the inductor to be distributed between the two outputs in a time-multiplexed fashion. Dead-time generators are used to eliminate shoot-through current by ensuring that $S_{1}$ and $S_{2}$ are not turned ON simultaneously. Dead-times are also introduced between $S_{a}$ and $S_{b}$ to prevent inadvertent cross conduction between the two LED strings.

Since an LED is essentially a current driven device, an LED driver typically regulates the LED current rather than its forward voltage. A straightforward way is to insert a small high-precision current sensing resistor $\left(R_{\mathrm{csa}}, R_{\mathrm{csb}}\right)$ in series with the corresponding LED string to sense the LED current by converting it to the current-sense voltage $\left(V_{\mathrm{csa}}, V_{\mathrm{csb}}\right)$. The current-sense voltage is then compared with the reference voltage $\left(V_{\mathrm{refa}}, V_{\mathrm{refb}}\right)$ to generate the corresponding logic signals (outa_req, outb_req) which determine the opening or closing of the two output switches in a SIDO buck converter. Since the LED's $I-V$ curve is usually provided by the LED manufacturer, the target dc current value for a particular LED string can be set by choosing an appropriate reference voltage. On the other hand, a two-limit hysteretic control determines the on-time of the high-side and low-side power switches $\left(S_{1}, S_{2}\right)$ of the buck converter. The upper and lower limits of the inductor current, namely the peak current limit and the valley current limit, define the average value of the inductor current which is the total LED current for a SIDO buck LED driver. In DCM, the valley current limit is set to zero to prevent the inductor current from going negative which degrades the power conversion efficiency [12], [16], [25]. As illustrated in Fig. $1, R_{f} \mathrm{C}_{f}$ is connected in parallel to the inductor so that the slopes of $V_{R C}$ are proportional to the inductor current ramp-up and ramp-down slopes [26]. A small resistor ladder is connected between $V_{R C}$ and ground in order to generate a lower voltage signal $V_{i L \text { sense }}$ which falls within the input voltage range of the comparator (CMP). $V_{0 i L \text { sense }}$ is fed forward to the corresponding comparators to determine the peak-crossing and zero-crossing of the inductor current. Fig. 2 is a simplified flowchart showing the system-level operation of the proposed SIDO buck driver. Suppose identical current flows through each of the two LED strings, also referred to as the balanced load condition, the inductor current $I_{L}$ is assigned to each string in alternate switching cycles. The working principle of the proposed SIDO buck LED driver is represented by the timing diagram shown in Fig. 3. During $D_{1 a} T_{s}$ or $D_{1 b} T_{s}, I_{L}$ ramps up with a slope of $m_{1}=\left(V_{g}-V_{o}\right) / L$ and the inductor is charged with a voltage of $V_{L}=V_{g}-V_{o}$, where $V_{g}$ and $V_{o}$ represent the input voltage and the output voltage, respectively. During $D_{2 a} T_{s}$ or $D_{2 b} T_{s}, I_{L}$ ramps down with a slope of $m_{2}=$ $-V_{o} / L$ and the inductor discharges its current to the corresponding output capacitor and the LED string until $I_{L}$ returns to zero. During $D_{3 a} T_{s}$ or $D_{3 b} T_{s}, I_{L}$ stays at zero with both $S_{1}$ and $S_{2}$ OFF. In the proposed SIDO LED driver, the system clock defines the switching frequency. The rising edge of the system clock triggers the ON duty cycle $\left(D_{1 a} T_{s}, D_{1} b T_{s}\right)$ by charging up the inductor during which $S_{1}$ is ON and $S_{2}$ is OFF. The 


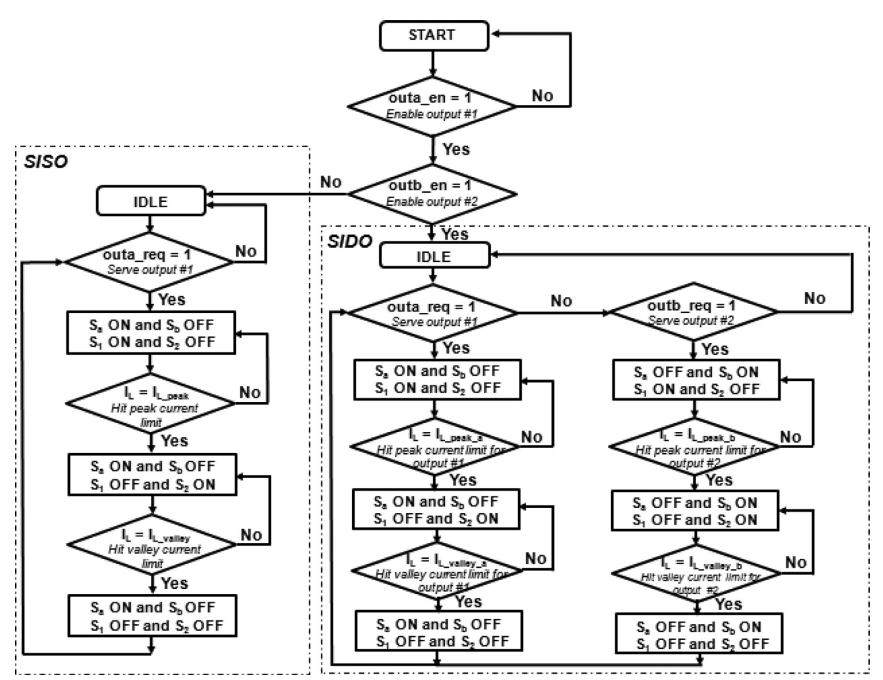

Fig. 2. Simplified flowchart representing the system-level operation of the proposed SIDO buck LED driver.

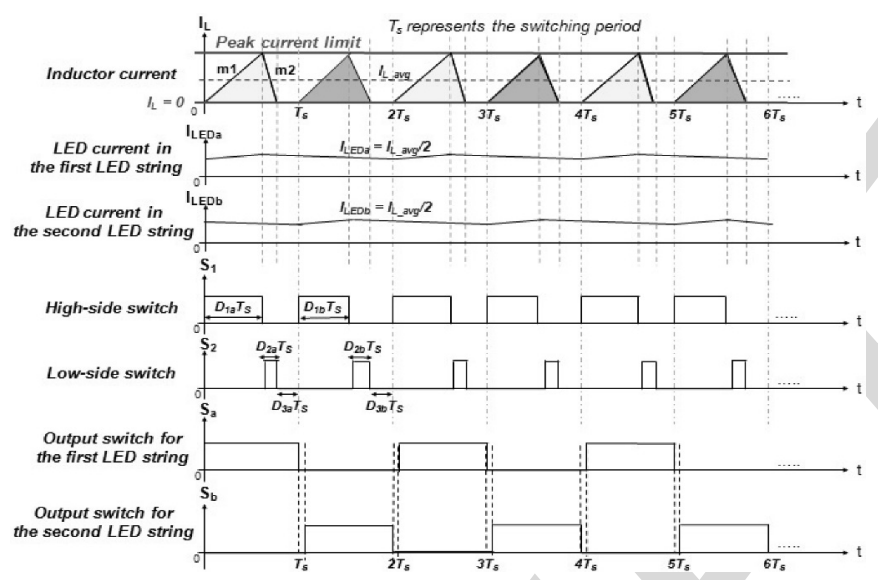

Fig. 3. Timing diagram of the proposed SIDO buck LED driver with balanced load operating in DCM. inductor current continues to increase until it hits the peak current limit at which point the buck converter enters $\left(D_{2 a} T_{s}, D_{2 b} T_{s}\right)$ where $S_{1}$ is OFF and $S_{2}$ is ON. The inductor discharges its current to the corresponding output until the zero-crossing of the inductor current is detected. The converter then enters the idle phase $\left(D_{3 a} T_{s}, D_{3 b} T_{s}\right)$ during which both $S_{1}$ and $S_{2}$ are OFF. The inductor current remains at zero until the next rising edge of the system clock arrives and the switching sequence repeats itself. The two output switches $\left(S_{a}, S_{b}\right)$ are controlled by the FSM as shown in Fig. 4.

The state machine is triggered by the rising edge of the system clock (sysclk) so that the transitions of the output switches $\left(S_{a}, S_{b}\right)$ are in sync with the system clock. The input signals of the state machine are the output enable signals (outa_en, outb_en) and the output request signals (outa_req, outb_req) which determine the switching sequence of the two outputs. The first LED string is always given a higher priority over the second one. For instance, if both strings request service simultaneously, i.e., outa_req $=1$ and $o u t b \_r e q=1, S_{a}$ is turned ON first and $S_{b}$ remains OFF. $S_{b}$ is turned ON only when outa_req $=0$

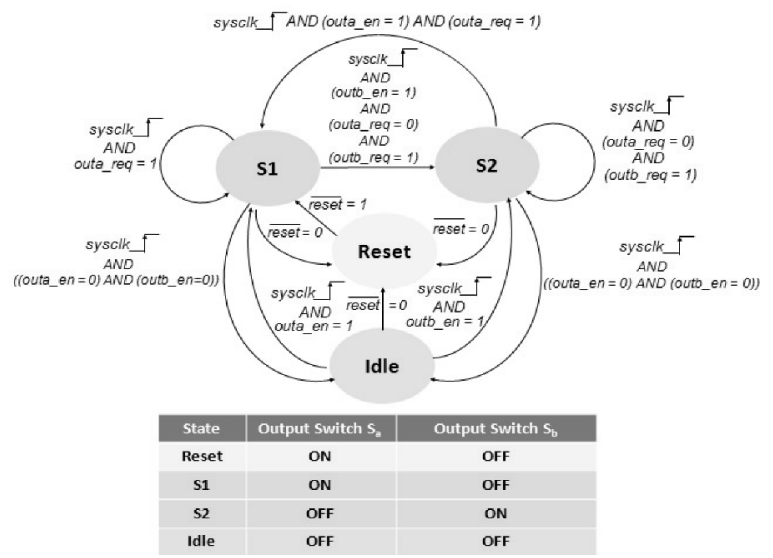

Fig. 4. State diagram of the proposed FSM for controlling the two output switches in SIDO buck LED driver.

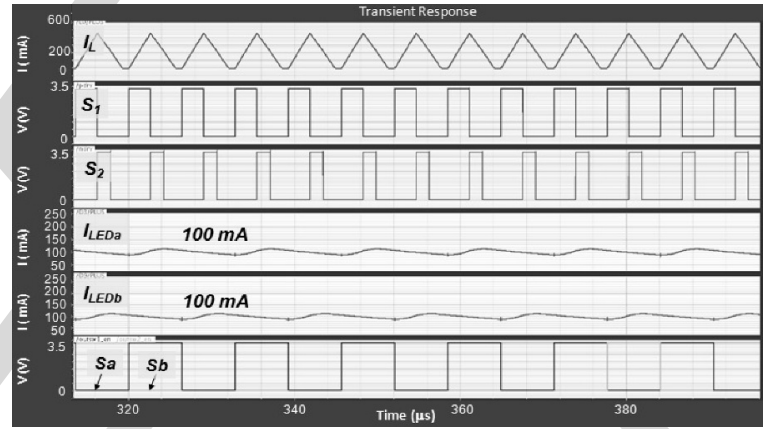

Fig. 5. Simulated steady-state waveforms for the proposed SIDO buck LED driver operating in DCM.

and $o u t b \_r e q=1 . S_{a}$ and $S_{b}$ must be nonoverlapping to avoid undesirable cross conduction between the two LED strings. In addition, an enable signal (outlen, out2en) is associated with either of the two LED strings. It provides the option of shutting down any or all of the LED strings, for example, in response to an overcurrent fault condition. An overriding signal can also be sent from the FSM to the hysteretic controller to disable the high-side and low-side power switches accordingly. The FSM-based controller can be modified quickly and conveniently to drive multiple LED strings in a SIMO configuration by simply adding more states in the VHDL or Verilog code. A mixed-signal macromodel of the proposed FSM-based digitally controlled SIDO buck switching LED operating in DCM is simulated in the time domain using Cadence Spectre [27]. The FSM is modeled in Verilog RTL and the rest are modeled as ideal circuit elements. The simulation model also incorporates parasitics such as DCR of the inductor $L$ and ESR of the output capacitors $\left(C_{o a}, C_{o b}\right)$. For balanced load condition, the current between the two LED strings is identical and each string consists of two LEDs connected in series. First, the steadystate performance is investigated. Fig. 5 contains the simulated steady-state waveforms for the inductor current $\left(I_{L}\right)$, the LED current $\left(I_{\mathrm{LED} a}, I_{\mathrm{LED} b}\right)$, and the four switches $\left(S_{1}, S_{2}, S_{a}, S_{b}\right)$ of the proposed SIDO buck LED driver operating in DCM. The switching frequency is $156.25 \mathrm{kHz}$ and the input voltage $V_{g}$ is $15 \mathrm{~V}$. The simulation results show that the LED current in either 


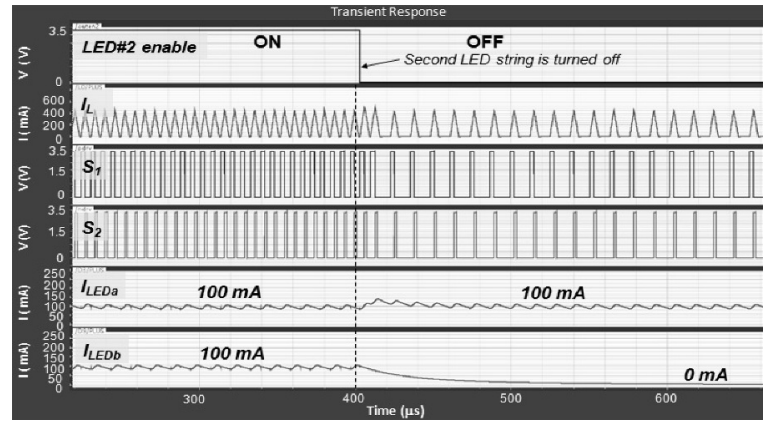

Fig. 6. First LED string remains under regulation without cross regulation when the second LED string is shut down completely.

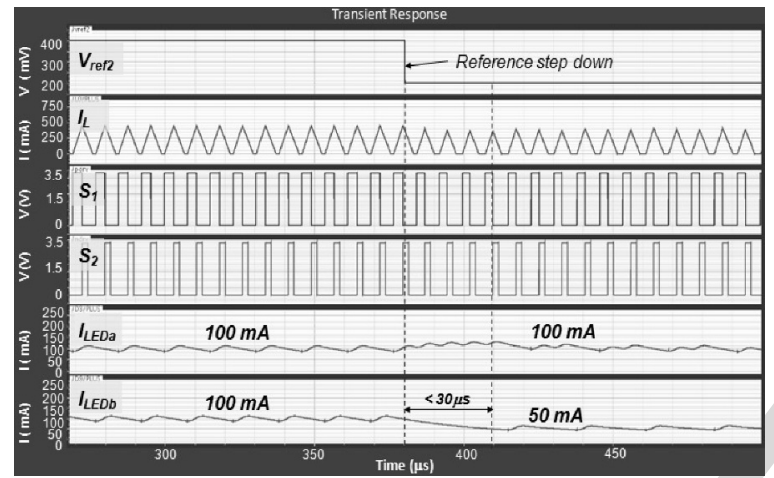

Fig. 7. First LED string remains under regulation without cross regulation despite a reference step in the second LED string from 100 to $50 \mathrm{~mA}$ in $20 \mathrm{~ns}$.

of the two strings is regulated successfully to the target steadystate dc value of $100 \mathrm{~mA}$ with a current ripple of $23 \backslash \%_{\mathrm{P}-\mathrm{P}}$. The steady-state output voltage for the first and second LED string is approximately $6.48 \mathrm{~V}$ with a voltage ripple of $2.6 \%$ P-P. Second, the stability of the closed-loop system is verified by examining its dynamic performance. In the first scenario, the second LED string needs to be shut down instantly in response to an over-current condition. Fig. 6 shows that despite the immediate shutdown of the second LED string, the LED current $I_{\mathrm{LED} a}$ in the first LED string continues to be regulated successfully at its target nominal value of $100 \mathrm{~mA}$ with minimal cross regulation. In the second scenario, the second LED string experiences a reference step of $50 \mathrm{~mA}$, i.e., $I_{\mathrm{LED} b}$ transitions from 100 to $50 \mathrm{~mA}$ in $20 \mathrm{~ns}$. Fig. 7 shows that the current in the first LED string continues to be regulated at around $100 \mathrm{~mA}$, virtually unaffected by the sudden reference step in the other string. The second LED string settles to the new nominal current value of $50 \mathrm{~mA}$. It demonstrates that the closed-loop system remains stable in response to the reference transient in the second string.

Unlike conventional backlight LED drivers that use PWM dimming transistor connected in series with the LED string [3], [8], [28]-[31], the proposed SIDO LED driver takes advantage of the existing four switches to perform dimming without requiring additional switches. When the dimming control signal for a particular LED string goes high, certain phases of the inductor current are skipped so that the average inductor current (also the average load current) going into that string is reduced accordingly. The digital dimming control signals (dim_ctrll,

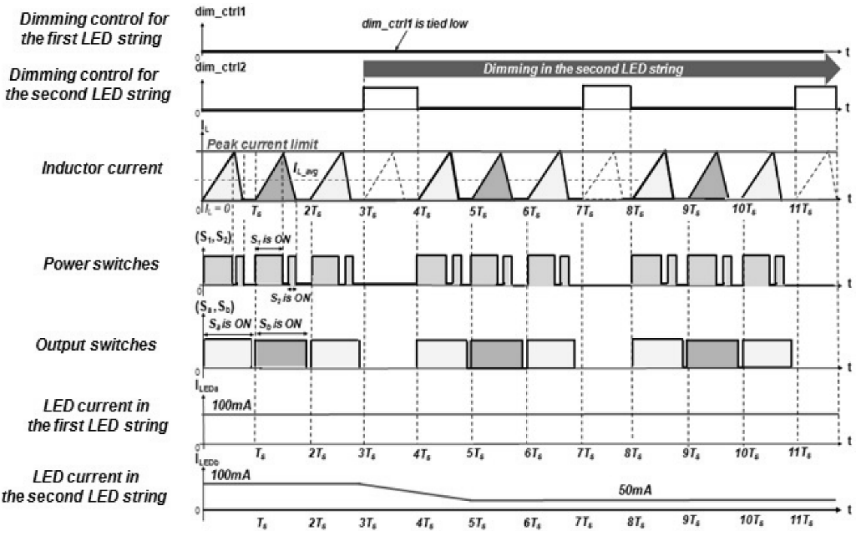

Fig. 8. Proposed digital dimming control in SIDO buck LED driver.

dim_ctrl2) essentially modulate the dc current level flowing 255 through the corresponding LED string. No additional dimming 256 transistors in series with the LED string are required, thereby 257 leading to a smaller voltage headroom and reduced power loss. 258 The only voltage headroom is the voltage across the current- 259 sense resistor $\left(V_{\text {csa }}, V_{\text {csb }}\right)$ which is typically between 0.2 and 260 $0.4 \mathrm{~V}$. Fig. 8 depicts the timing diagram of the proposed digital 261 dimming control scheme. In this particular case, the second LED 262 string is dimmed by reducing its current from 100 to $50 \mathrm{~mA}, 263$ while the current in the first LED string stays constant at $100 \mathrm{~mA} .264$

Any combination of LED strings in a SIMO LED driver can 265 be dimmed or even shut down momentarily to achieve flexi- 266 ble dimming and optimum luminance levels. In addition, it is 267 reported in the literature [32]-[34] that a bilevel or $N$-level cur- 268 rent driving technique for LED dimming improves the luminous 269 efficacy of LEDs by introducing a dc offset to the PWM cur- 270 rent. The proposed SIDO converter can potentially be used as 271 a bilevel LED driver by generating two programmable dc cur- 272 rent values for each individual LED string in a time-multiplexed 273 fashion. Another major difference between the proposed LED 274 driver and the existing ones [3], [8], [28]-[31] is that the former 275 provides $N$ optimized output bus voltage for each individual 276 LED string, whereas the latter only uses a common output bus 277 shared by all the LED strings. Due to manufacture, process, 278 and temperature variations, $V_{F}$ in each LED does not match 279 perfectly, which means that the voltage drop across each LED 280 string differs. Using the proposed SIDO buck LED driver in 281 Fig. 1 as an example and assuming the LED current is $100 \mathrm{~mA} 282$ in each string, the voltage headroom $\left(V_{\mathrm{csa}}, V_{\mathrm{csb}}\right)$ is $0.4 \mathrm{~V}$, and 283 the voltage drop across each of the two LED strings are $V_{F a}=284$ $6.0 \mathrm{~V}$ and $V_{F b}=7.0 \mathrm{~V}$, respectively. The output voltages are 285 $V_{o a}=6.4 \mathrm{~V}$ and $V_{o b}=7.4 \mathrm{~V}$. The total power consumption 286 of the load $P_{\mathrm{LOAD}}$, including the LED string and current-sense 287 resistor, is $P_{\mathrm{LOAD}}=V_{o a} \times I_{\mathrm{LED}}+V_{o b} \times I_{\mathrm{LED}}=1.38 \mathrm{~W}$. The 288 output voltage for each LED string is independently optimized 289 based on its corresponding $V_{F}$, resulting in the same voltage 290 headroom of $0.4 \mathrm{~V}$ for each string. This is different from a con- 291 ventional LED driver in which the common output bus voltage 292 is usually regulated using the LED string with the maximum 293 voltage drop. For the same LED current, the total power con- 294 sumption using a conventional LED driver is given by: $P_{\mathrm{LOAD}}=295$ 


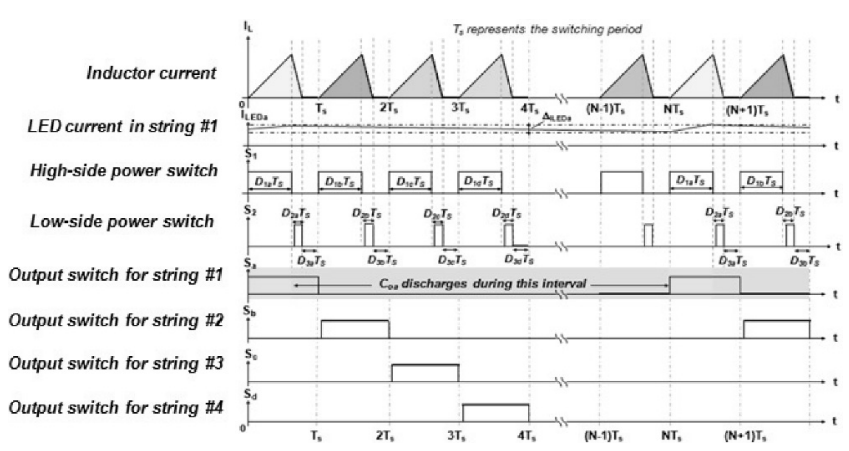

Fig. 9. Timing diagram of the proposed SIMO buck LED driver.

$2 \times \max \left(V_{o a}, V_{o b}\right) \times I_{\mathrm{LED}}=1.48 \mathrm{~W}$, which is more than $7 \%$ higher than that of the proposed driver. The voltage headroom for the first LED string increases from 0.4 to $1.4 \mathrm{~V}$, resulting in $450 \mathrm{~mW}$ more power loss or additional $30 \%$ efficiency degradation. Since the output voltage is self-optimized to match the total $V_{F}$ in each individual LED string in the proposed driver, same-colored LEDs from neighboring bins (not only from a single bin) with larger $V_{F}$ variance can be used which helps reduce the LED costs. In the event that a particular application demands a total LED current greater than the average inductor current, the same time-multiplexing control scheme operating in DCM can still be employed either by lowering the switching frequency with a higher inductor peak current limit or by operating the buck LED driver in pseudocontinuous conduction mode (PCCM) [17], [35], [36]. In PCCM, the average inductor current is increased by simply adding a nonzero dc offset of $I_{\mathrm{DC}}$ to that of DCM.

\section{EXTENSION FROM SIDO TO SIMO BUCK LED DRIVER}

Having demonstrated the feasibility of the proposed SIDO buck LED driver, it is natural for us to extend it to SIMO with $N$ independently driven LED strings. In particular, the theoretical maximum number of LED strings $N_{\max }$ is determined for this SIMO architecture. Fig. 9 shows a timing diagram of the inductor current, the two power switches $\left(S_{1}, S_{2}\right)$, and the first four output switches $\left(S_{a}, S_{b}, S_{c}, S_{d}\right)$ in a SIMO buck LED driver. To simplify the analysis, the balanced load condition is assumed. Based on the time-multiplexing control scheme, energy is being transferred from the dc supply to each individual output exactly once within a total of $N$ switching phases. For a particular output, the corresponding output switch is OFF during $D_{3}$, while the output capacitor discharges to the LED string. During the subsequent $(N-1) \times T_{s}$ phases, the output switch remains OFF and the output capacitor continues to discharge to the corresponding LED string. Hence, the total discharging time for the output capacitor $t_{\mathrm{dch}}$ can be expressed as

$$
\begin{aligned}
t_{\mathrm{dch}} & =D_{3} T_{s}+(N-1) T_{s}=\left(D_{3}+N-1\right) T_{s} . \\
\text { For } D_{3} & =0, t_{\mathrm{dch}}=(N-1) T_{s} .
\end{aligned}
$$

The proposed SIMO buck LED driver is essentially a constant-current regulator which maintains a constant dc current $I_{\mathrm{LED}}$ flowing through the LED string via a closed-loop current-sense feedback control. For very small variation of forward voltage around the quiescent point (also known as bias point) on the LED's exponential $I-V$ curve, the de forward current is assumed to be constant. During $t_{\mathrm{dch}}$ when the output switch is OFF, the output capacitor is connected to the LED string which acts as a constant-current sink (CCS). Assuming ideal capacitor with no ESR (the effect of the ESR will be explained later), the voltage across the output capacitor $v_{c}(\mathrm{t})$ is the same as the output voltage which is expressed as the charge $q(t)$ divided by the capacitance value $C_{o}$, i.e.,

$$
v_{c}(t)=\frac{q(t)}{C_{o}}=\frac{1}{C_{o}} \int_{0}^{t_{\mathrm{dch}}} i_{c}(\tau) d \tau+v_{c}(0)
$$

For CCS, $i_{c}(\tau)=I_{\mathrm{LED}}$.

Combining (3) and (4) and rearranging, we have

$$
\Delta v_{o}=\Delta v_{c}=v_{c}(t)-v_{c}(0)=\frac{1}{C_{o}}\left(I_{\mathrm{LED}} t_{\mathrm{dch}}\right) .
$$

Hence, the total discharging time $t_{\mathrm{dch}}$ can be expressed as

$$
t_{\mathrm{dch}}=\frac{C_{o} \Delta v_{o}}{I_{\mathrm{LED}}}
$$

where $\Delta v_{o}$ is the output voltage drop due to the discharging 346 of the output capacitor. In general, $\Delta v_{o}$ is assumed to be rea- 347 sonably small relative to the output voltage. The LED ripple 348 current $\Delta i_{\text {LED }}$ usually ranges from $10 \%$ P-P to $40 \%$ P-P of the 349 dc forward current as recommended by the LED manufactur- 350 ers [37], [38]. For a particular $\Delta i_{\mathrm{LED}}$, the corresponding voltage 351 ripple $\Delta v_{\mathrm{LED}}$ at the chosen bias point can be readily obtained 352 from the exponential $I-V$ curve. Suppose each LED string con- 353 tains a total of $n$ LEDs connected in series. The output voltage 354 ripple $\Delta v_{o}$ is, therefore, the sum of the voltage ripple across 355 the LED string and the voltage ripple across the current-sense 356 resistor, i.e., $\Delta v_{o}=n \times \Delta v_{\mathrm{LED}}+\Delta v_{c s}$. Suppose $\Delta v_{\text {omax }}$ rep- 357 resents the maximum output voltage ripple allowed. Equation (6) 358 can, therefore, be rewritten as

$$
t_{\mathrm{dch}} \leq \frac{C_{o} \Delta v_{o \text { max }}}{I_{\mathrm{LED}}} .
$$

Substituting (1) into (7), we have

$$
\begin{aligned}
\left(D_{3}+N-1\right) T_{s} & \leq \frac{C_{o} \Delta v_{o \max }}{I_{\mathrm{LED}}} \Rightarrow N \\
& \leq \frac{C_{o} \Delta v_{o \text { max }}}{I_{\mathrm{LED}} T_{s}}+1-D_{3} .
\end{aligned}
$$

Hence, the theoretical maximum possible number of LED 361 strings in SIMO, $N_{\max }$, is given by

$$
N_{\max }=\frac{C_{o} \Delta v_{o \text { max }}}{I_{\mathrm{LED}} T_{s}}+1-D_{3}=\frac{C_{o} \Delta v_{o \text { max }}}{I_{\mathrm{LED}} T_{s}}+D_{1}+D_{2} .
$$

Since $N_{\max }$ is an integer value, the $\operatorname{floor}(\cdot)$ function is used to round the result down to the closest integer. Hence, (9a) becomes

$$
N_{\max }=\text { floor }\left(\frac{C_{o} \Delta v_{o \text { max }}+I_{\mathrm{LED}} T_{s}\left(1-D_{3}\right)}{I_{\mathrm{LED}} T_{s}}\right) .
$$

Equation (9b) represents a general formula for determining the 365 scalability limit of a SIMO buck LED driver operating in DCM 366

.

.

.

49

350

351

352

53

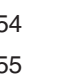

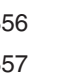


and is referred to as a scalable DCM-based SIMO scheme for the sake of our ensuing discussion. In particular, when $D_{3}=$ 0 , the SIMO buck LED driver operates in boundary conduction mode (BCM). Hence, (9a) and (9b) become (10a) and (10b), respectively. Also, $N_{\max }$ in BCM is greater than or equal to that in DCM for the same set of design parameter values

$$
\begin{aligned}
& N_{\max }=\frac{C_{o} \Delta v_{o \text { max }}}{I_{\mathrm{LED}} T_{s}}+1 \\
& N_{\max }=\text { floor }\left(\frac{C_{o} \Delta v_{o \text { max }}}{I_{\mathrm{LED}} T_{s}}+1\right) .
\end{aligned}
$$

For a single-output buck converter, the average inductor current is identical to the load current. Due to the nature of the timemultiplexing control scheme in the proposed SIMO converter, the average inductor current $I_{L_{-} \text {avg }}$ is the sum of the individual load current $I_{\mathrm{LED}}$ in each LED string. Assuming balanced load condition, $I_{L_{-} \text {avg }}=N \times I_{\mathrm{LED}}$, where $N$ is the total number of LED strings. The average inductor current reaches its maximum value in BCM, resulting in a maximum transfer of power [16]. Since the current in each LED string remains the same, a theoretical upper bound of the total achievable number of LED strings in SIMO can be expressed as

$$
N_{\max }=\frac{I_{L_{-} \text {avg_max }}}{I_{\mathrm{LED}}} .
$$

By simple geometry, $I_{L_{-} \text {avg_max }}$ is given by the following equation [39]:

$$
I_{L_{-} \text {avg_max }}=\frac{m_{1} D_{1} T_{s}}{2}=\frac{\left(V_{g}-V_{o}\right) D_{1} T_{s}}{2 L} .
$$

By substituting (12) into (11) and rearranging, $T_{s}$ can be expressed as

$$
T_{s}=\frac{2 L N_{\max } I_{\mathrm{LED}}}{D_{1}\left(V_{g}-V_{o}\right)} .
$$

Now, by substituting (13) into (10a) and rearranging, we have $2 L I_{\mathrm{LED}}^{2} N_{\mathrm{max}}^{2}-2 L I_{\mathrm{LED}}^{2} N_{\max }-C_{o} \Delta v_{o \max }\left(V_{g}-V_{o}\right) D_{1}=0$.

Equation (14) is a quadratic equation in $N_{\max }$. The discriminant $\Delta$ of (14) can be expressed as

$$
\Delta=4 L^{2} I_{\mathrm{LED}}^{4}+8 L I_{\mathrm{LED}}^{2} C_{o} \Delta v_{o \max }\left(V_{g}-V_{o}\right) D_{1}>0 .
$$

Since $\left(V_{g}-V_{o}\right)>0$ for a buck switcher, the discriminant in (15) is always a positive number which implies that (14) has two real roots as given by

$$
r_{1}, r_{2}=\frac{2 L I_{\mathrm{LED}}^{2} \pm \sqrt{\Delta}}{4 L I_{\mathrm{LED}}^{2}} .
$$

Since $N_{\max }$ must be a positive integer, the negative root is eliminated, leaving only the positive root, i.e.,

$$
\begin{aligned}
& N_{\max \_ \text {BCM }} \\
& =\text { floor }\left(\frac{1}{2} \times\left[1+\sqrt{1+\frac{2 C_{o} \Delta v_{o \text { max }} V_{o}\left(V_{g}-V_{o}\right)}{L I_{\mathrm{LED}}^{2} V_{g}}}\right]\right)
\end{aligned}
$$

Equation (17) defines the theoretical maximum total number of outputs in SIMO operating in BCM. It is referred to as a scalable BCM-based SIMO scheme which is a special case of 398 scalable DCM-based SIMO scheme. In fact, it is observed that (11) is also valid for the case of DCM. By simple geometry, the switching period $T_{s}$ in DCM can be expressed as

$$
T_{s}=\frac{2 L N_{\max } I_{\mathrm{LED}}}{D_{1}\left(D_{1}+D_{2}\right)\left(V_{g}-V_{o}\right)} .
$$

Realizing that the same calculations that lead to (17) for the 402 case of BCM can also be performed in DCM, the theoretical 403 maximum total number of LED strings in a SIMO converter 404 operating in DCM can, therefore, be written as ${ }^{1}$

$$
\begin{aligned}
N_{\text {max _DCM }} & =\text { floor }\left(\frac{1}{2} \times\left(1-D_{3}\right)\right. \\
\times & {\left.\left[1+\sqrt{1+\frac{2 C_{o} \Delta v_{o \max }\left(V_{g}-V_{o}\right) D_{1}}{L I_{\mathrm{LED}}^{2}\left(1-D_{3}\right)}}\right]\right) . }
\end{aligned}
$$

Notice that for the case of BCM, $D_{3}=0$ and $D_{1}=V_{o} / V_{g}, 406$ (19) reduces to (17). Hence, (19) represents the generalized 407 formula for the theoretical maximum total number of outputs 408 in SIMO which is applicable to either BCM or DCM. It is also 409 interesting to note that the average inductor current in DCM 410 is smaller than (or equal to) that in BCM. As a result, for the 411 same LED current, the theoretical maximum achievable number 412 of outputs in SIMO operating in DCM is no greater than that 413 in BCM, i.e., $N_{\max \_ \text {DCM }} \leq N_{\text {max_BCM }}$. In reality, the ESR of 414 the output capacitor needs to be taken into consideration. Any 415 current flowing through the output capacitor $C_{o}$ must also flow 416 through the $R_{\mathrm{ESR}}$, resulting in an additional voltage drop of 417 $\Delta V_{\mathrm{ESR}}=I_{\mathrm{LED}} \times R_{\mathrm{ESR}}$. Hence, $\Delta v_{o}$ can be expressed as

$$
\Delta v_{o}=\Delta v_{c}+\Delta v_{\mathrm{ESR}}=\Delta v_{c}+I_{\mathrm{LED}} \times R_{\mathrm{ESR}} .
$$

Rearranging the terms in (20), we have

$$
\Delta v_{c}=\Delta v_{o}-I_{\mathrm{LED}} \times R_{\mathrm{ESR}} .
$$

Hence, (17) and (19) are modified slightly to become (22) and 420 (23), respectively: BCM:

$$
\begin{gathered}
N_{\text {max } \_ \text {BCM }}=\text { floor }\left(\frac{1}{2} \times[1\right. \\
\left.+\sqrt{\left.1+\frac{2 C_{o} V_{o}\left(\Delta v_{o \text { max }}-I_{\mathrm{LED}} R_{\mathrm{ESR}}\right)\left(V_{g}-V_{o}\right)}{L I_{\mathrm{LED}}^{2} V_{g}}\right]}\right)
\end{gathered}
$$

DCM:

$$
\begin{aligned}
& N_{\text {max _DCM }}=\text { floor }\left(\frac{1}{2} \times\left(1-D_{3}\right) \times[1\right. \\
& \left.\left.+\sqrt{1+\frac{2 C_{o}\left(\Delta v_{o \text { max }}-I_{\mathrm{LED}} R_{\mathrm{ESR}}\right)\left(V_{g}-V_{o}\right) D_{1}}{L I_{\mathrm{LED}}^{2}\left(1-D_{3}\right)}}\right]\right) .
\end{aligned}
$$

The presence of $R_{\mathrm{ESR}}$ in (22) and (23) reduces the theoretical 423 maximum achievable number of outputs in SIMO. Therefore, 424

\footnotetext{
${ }^{1}$ In DCM, $D_{1}$ can be expressed as: $D_{1}=M \sqrt{\frac{K}{1-M}}$, where $M=\frac{V_{o}}{V_{q}}$ and $K=\frac{2 L}{R_{L} T_{s}}=\frac{2 L I_{\mathrm{LED}}}{V_{o} T_{s}}[39]$.
} 


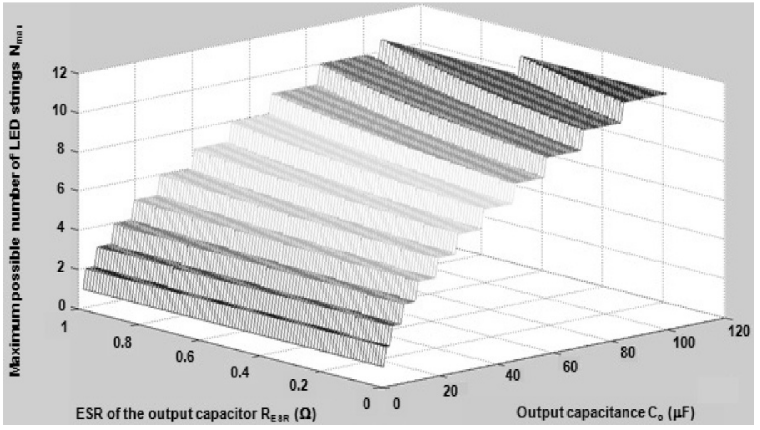

Fig. 10. Theoretical maximum achievable number of LED strings $\left(N_{\max }\right)$ versus the output capacitance $\left(C_{o}\right)$ and the capacitor $\operatorname{ESR}\left(R_{\mathrm{ESR}}\right)$ for the scalable BCM-based SIMO scheme. it is always recommended to choose an output capacitor with a smaller ESR, whenever possible. Fig. 10 shows the theoretical maximum total number of LED strings versus the output capacitance and capacitor ESR for the scalable BCM-based SIMO scheme, given an LED current of $80 \mathrm{~mA}$ and a maximum ripple current requirement of $40 \%$ P-P.

Intuitively, for a particular LED current, an increasing number of outputs can be achieved by using a larger output capacitor with the same ESR value. For instance, if the output capacitance is increased from 4.7 to $22 \mu \mathrm{F}$ (the ESR remains at $100 \mathrm{~m} \Omega$ ), the theoretical model based on (22) suggests that the maximum total number of LED strings can be increased from three to six. It is also interesting to note that the maximum number of outputs in SIMO has a stronger dependence on the output capacitance than the capacitor ESR, as shown in Fig. 10.

On the other hand, it is useful to study how the LED current affects the maximum achievable number of outputs in SIMO. As an example, assuming balanced load and two LEDs connected in series per string, a scalable BCM-based SIMO scheme is investigated with these parameter values: $L=47 \mu \mathrm{H}, C_{o}=$ $4.7 \mu \mathrm{F}, R_{\mathrm{ESR}}=100 \mathrm{~m} \Omega, V_{g}=15 \mathrm{~V}$, and $V_{o}=6.4 \mathrm{~V}$. The relationship between $N_{\max }$ and $I_{\mathrm{LED}}$ can be obtained by using (22) for different values of output voltage ripple $\Delta v_{o \_ \text {max }}$. Based upon the $I-V$ curve and/or SPICE model of the particular LED used, the corresponding output voltage ripple $\Delta v_{o_{-} \max }$ can be determined from the LED current ripple requirement $\Delta i_{\mathrm{LED}}$. The proposed design uses white LED [40] which is the target for LCD backlighting applications. For instance, a 20\%p-p current ripple corresponds to around 2\% 2 -P voltage ripple and a $40 \%$ P-P current ripple corresponds to around 4\% -P voltage ripple. Fig. 11 shows a plot of $N_{\max }$ versus $I_{\mathrm{LED}}$ for $\Delta i_{\mathrm{LED}}$ ranging from 5\%p-p to $40 \%$ p-P. This plot is beneficial to a practical SIMO design in two ways. First, for a given LED current and current ripple requirement, the theoretical maximum number of LED strings viable under the scalable BCM-based SIMO scheme can be extracted directly from the plot. Second, the

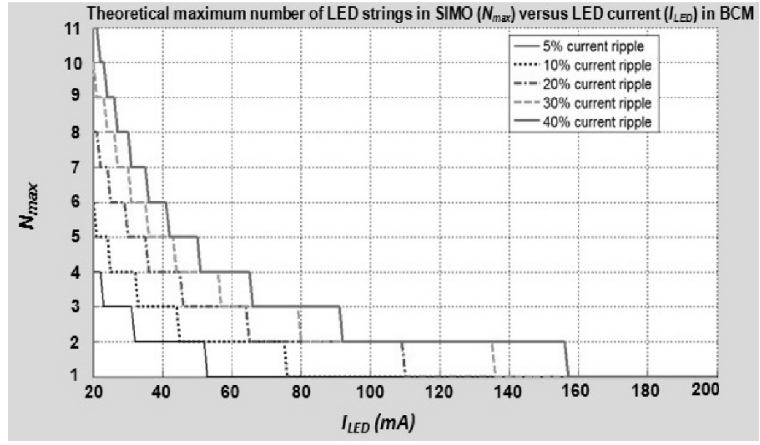

Fig. 11. Plot of theoretical maximum number of LED strings in SIMO $\left(N_{\mathrm{max}}\right)$ versus the LED current $\left(I_{\mathrm{LED}}\right)$ in the scalable BCM-based SIMO scheme.

maximum LED current allowed in order for a SIMO to remain 461 at the same scaling level can also be obtained from the plot. For 462 instance, given a $20 \%$ current ripple requirement (i.e., $\Delta i_{\mathrm{LED}}=463$ $20 \%$ p-P), a SIDO (dual-string) configuration is possible as long 464 as the LED current in each string is no more than $110 \mathrm{~mA}$. In 465 the event that an application demands an LED current greater 466 than $110 \mathrm{~mA}$, two options can be considered: 1) Relax the cur- 467 rent ripple requirement whenever possible. A wider tolerance 468 in $\Delta i_{\mathrm{LED}}$ is generally acceptable since the ripple frequency is 469 too high for the human eye to detect. 2) Operate the SIMO buck 470 LED driver in PCCM [17], [35], [36]. In PCCM, the floor of 471 the inductor current is raised by a nonzero dc offset $I_{\mathrm{DC}}$ which $\quad 472$ distinguishes it from DCM. The proposed theoretical model can 473 be extended to PCCM by adding a dc component to the aver- 474 age inductor current. By going through similar calculations as 475 in DCM, the theoretical maximum number of outputs in SIMO 476 operating in PCCM is given by (24), as shown at the bottom of 477 the page. It is interesting to note that (24) continues to apply to 478 the cases of DCM and BCM. For instance, in DCM, $I_{\mathrm{DC}}=0 \quad 479$ and (24), therefore, reduces to (23).

In the event of unbalanced load with unequal current among the LED strings, the scalable DCM- or BCM-based SIMO scheme continues to hold. The only change is to replace $I_{\mathrm{LED}}$ in (22) and (23) by $\max \left(I_{\mathrm{LED}}\right)$, where $\max \left(I_{\mathrm{LED}}\right)$ denotes the largest LED current among all the LED strings. In other words, the maximum number of LED strings that can be realized in a SIMO buck LED driver is constrained by the largest LED current. Generally speaking, the input voltage $V_{g}$, output voltage $V_{o}$, and the current ripple requirement are typically fixed parameters defined in the design specification. Without making any hardware changes (i.e., $L$ and $C_{o}$ values are fixed), the primary design variable in (22) and (23) is the LED current $I_{\mathrm{LED}}$. In fact, the LED current is the dominant factor for determining the maximum possible number of outputs under the scalable $D C M-/ B C M$-based SIMO scheme. By knowing the maximum LED current required for a particular application, the theoretical 1 62 463 464 465 66 467 168 9

470

1

472

3

474

45

46

47
48

$$
N_{\text {max } \_ \text {PCCM }}=\text { floor }\left(\frac{1}{2 I_{\mathrm{LED}}} \times\left[\left(I_{\mathrm{DC}}+\left(1-D_{3}\right) I_{\mathrm{LED}}\right] \times\left[1+\sqrt{\left.1+\frac{2 C_{o}\left(\Delta v_{o} \max \right.}{L\left[I_{\mathrm{LED}}-\left(1-D_{3}\right) I_{\mathrm{LED}}\right]^{2}}\right)\left(V_{g}-V_{o} D_{1}\left(1-D_{3}\right)\right.}\right]\right)\right.
$$


TABLE I

DESIGN SPECIFICATION OF A SISO BUCK LED DRIVER IN DCM

\begin{tabular}{|c|c|c|}
\hline Design Parameter & Value & Unit \\
\hline Input Voltage $\left(V_{g}\right)$ & 15 & $\mathrm{~V}$ \\
\hline Output Voltage $\left(V_{o}\right)$ & 6.32 & $\mathrm{~V}$ \\
\hline LED Forward Current $\left(I_{L E D}\right)$ & 80 & $\mathrm{~mA}$ \\
\hline Switching Frequency $\left(f_{s}\right)$ & 100 & $\mathrm{kHz}$ \\
\hline Inductor $(L)$ & 47 & $\mu \mathrm{H}$ \\
\hline Output Capacitor $\left(C_{o}\right)$ & 4.7 & $\mu F$ \\
\hline ESR of Output Capacitor $\left(R_{E S R}\right)$ & 100 & $\mathrm{~m} \Omega$ \\
\hline $\begin{array}{c}\text { Maximum LED Current Ripple } \\
\left(\Delta i_{L E D}\right)\end{array}$ & 40 & $\%_{\mathrm{P}-\mathrm{P}}$ \\
\hline $\begin{array}{c}\text { Maximum Output Voltage Ripple } \\
\left(\Delta v_{o}\right)\end{array}$ & 4 & $\%_{\mathrm{P}-\mathrm{P}}$ \\
\hline Duty Ratio of Idle Phase $\left(D_{3}\right)$ & $\geq 10$ & $\%$ \\
\hline
\end{tabular}

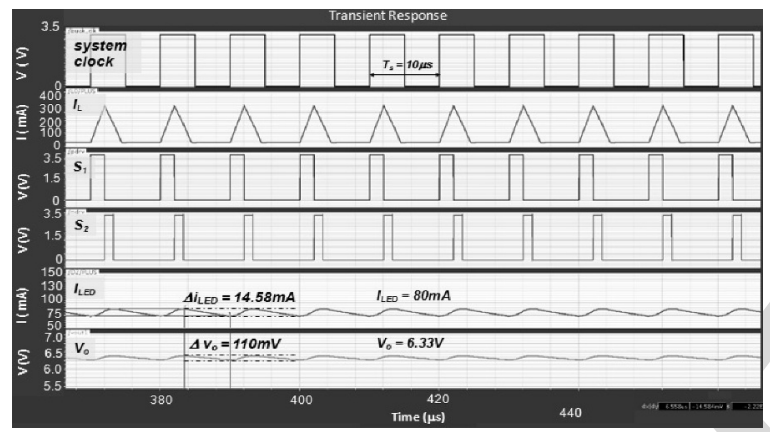

Fig. 12. Simulated steady-state waveforms for the SISO buck LED driver based on the design specification in Table I.

maximum achievable number of independently driven LED strings can be estimated in advance.

\section{Simulation Results}

Ideal macromodels based on the scalable DCM-based SIMO scheme were constructed and simulated in Cadence Spectre [27] in order to compare with the theoretical results in Section III. The design specification of a single-inductor single-output (SISO) buck converter is shown in Table I. The theoretical model based on (23) suggests that $N_{\text {max_DCM }}=1$, meaning only one LED string is viable. Fig. 12 shows the simulated steady-state waveforms of the inductor current $I_{L}$, the LED currents $I_{\mathrm{LED}}$, and the output voltages $V_{o}$ of a SISO buck LED driver. The simulated steady-state LED current $I_{\mathrm{LED}}$ is approximately $80 \mathrm{~mA}$ which meets the design target. The simulated LED current ripple $\Delta i_{\mathrm{LED}}$ is $18 \%$ P-P (also, the output voltage ripple $\Delta v_{o}$ is $1.7 \%$ p-p), which satisfies the maximum ripple requirement. Now, the SISO buck LED driver is transformed into SIDO by adding a second LED string. Fig. 13 shows the simulated steady-state waveforms from the resulting SIDO LED driver.

Despite the fact that the steady-state LED current in either string remains at $80 \mathrm{~mA}$, the LED current ripple is more than $40 \%$ p-p which violates the maximum ripple current requirement. Hence, the simulation results show that SIDO is not viable based on the design requirement which is consistent with the theoretical result. By increasing the switching frequency from

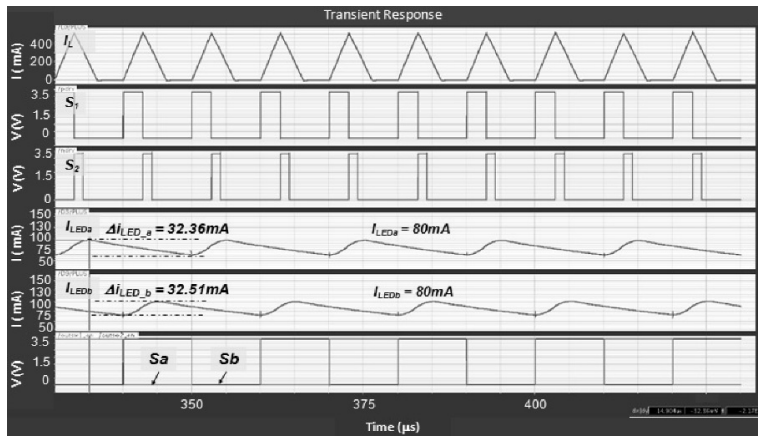

Fig. 13. Simulated steady-state waveforms showing SIDO is not viable since the $30 \%$ p-p maximum current ripple requirement is violated.

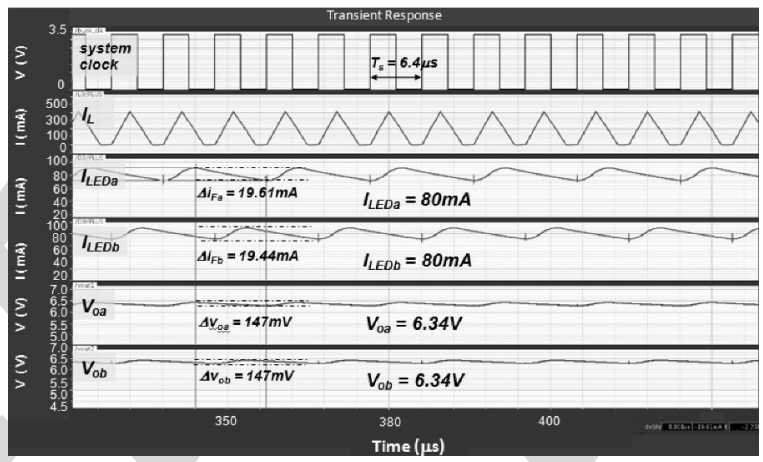

Fig. 14. Simulated steady-state waveforms showing SIDO is possible by increasing the switching frequency from 100 to $156.25 \mathrm{kHz}$

100 to $156.25 \mathrm{kHz}$ and keeping other parameters unchanged, 522 $N_{\text {max_DCM }}=2$ from (23). Fig. 14 shows the simulated wave- 523 forms for the corresponding signals in a SIDO buck LED driver. $\quad 524$ The simulated LED current ripple $\Delta i_{\mathrm{LED}}$ is $24 \%$ P-P and the cor- 525 responding output voltage ripple $\Delta v_{o}$ is $2.3 \%$ P-P , both of which 526 satisfy their corresponding maximum ripple requirement. Con- 527 sequently, both the theoretical and simulation results show that 528 by increasing the switching frequency, a SIDO buck LED driver 529 in DCM is feasible. 530

A third LED string is added to the SIDO buck LED driver to 531 transform it into SIMO consisting of three independently driven 532 LED strings. The LED current in each string remains unchanged 533 at $80 \mathrm{~mA}$ as in the SISO or SIDO case. According to Fig. 11, 534 the theoretical model suggests that for $I_{\mathrm{LED}}=80 \mathrm{~mA}$, a scal- 535 able BCM-based SIMO scheme with a maximum of three LED 536 strings is feasible under the $40 \%$ P-P current ripple constraint. 537 The switching period $T_{s}$ is chosen to be $6 \mu$ s using (13) which 538 corresponds to a switching frequency of $166.67 \mathrm{kHz}$. Fig. 15539 shows the simulated waveforms from the resulting SIMO buck 540 LED driver. The simulated LED current ripple $\Delta i_{\mathrm{LED}}$ is around 541 $35 \%$ p-P and the output voltage ripple $\Delta v_{o}$ is $3.5 \%$ P-P, both of 542 which satisfy their respective maximum ripple constraint. As a 543 sanity check, the theoretical model based on (22) indeed sug- 544 gests that a maximum possible number of three independently 545 driven LED strings can be achieved in the scalable BCM-based 546 SIMO scheme. Hence, it is shown that the simulation result 547 agrees with the corresponding theoretical result. On the other 548 hand, it is important to examine the transient performance of the 549 


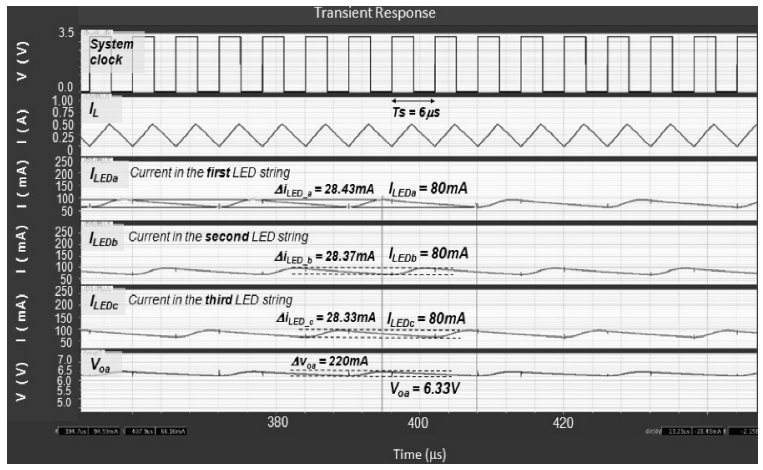

Fig. 15. Simulated steady-state waveforms of a three-string SIMO buck LED driver operating in $\mathrm{BCM}$

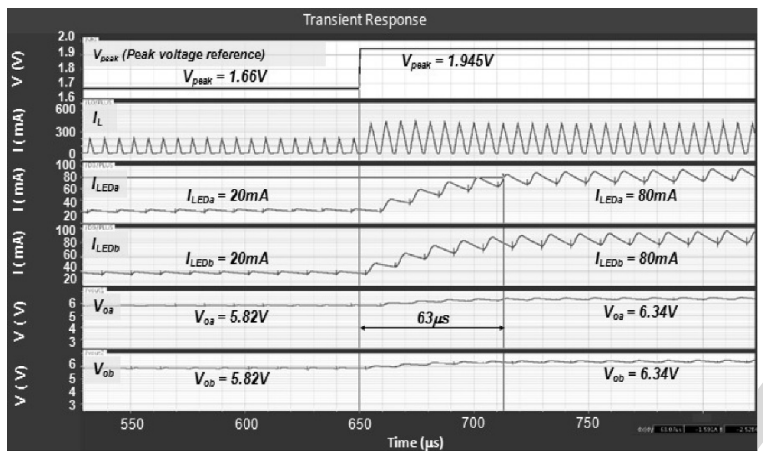

(a)

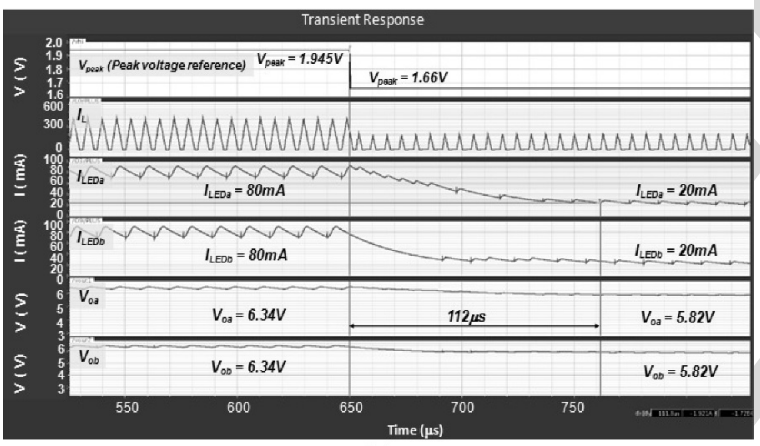

(b)

Fig. 16. Simulated transient waveforms for (a) peak reference step-up and (b) peak reference step-down response. proposed SIDO buck LED driver. The LED current is changed by adjusting the peak limit of the inductor current. By stepping up the peak voltage reference ( $V_{\text {peak }}$ in Fig. 1) from 1.660 to $1.945 \mathrm{~V}$ in $400 \mathrm{~ns}$, the peak inductor current limit is increased by approximately $200 \mathrm{~mA}$, leading to an increase in the nominal LED current from 20 to $80 \mathrm{~mA}$. The reference voltages $\left(V_{\text {ref } a}, V_{\text {ref } b}\right)$ are also stepped up from 100 to $340 \mathrm{mV}$ in order to maintain the same load current between the two LED strings. Conversely, by stepping down $V_{\text {peak }}$ from 1.945 to $1.660 \mathrm{~V}$, the LED current is reduced from 80 to $20 \mathrm{~mA}$. Fig. 16 shows the simulated transient behavior for the peak voltage reference step-up and step-down response.

In the case of step-up reference response, the LED current in either string settles to the steady-state nominal value of $80 \mathrm{~mA}$ within $63 \mu$ s. The output voltage reaches its target steady-state

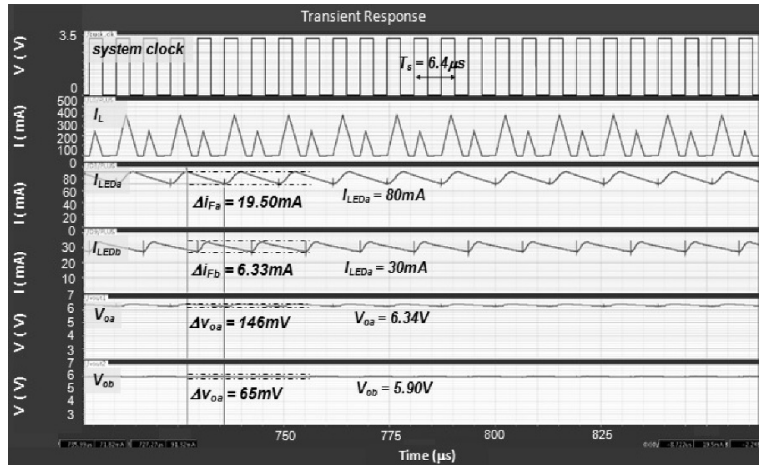

Fig. 17. Simulated steady-state waveforms of a SIDO buck LED driver with unbalanced load.

value of $6.34 \mathrm{~V}$. In the case of step-down reference response, the LED current in either string settles to the steady-state nominal value of $20 \mathrm{~mA}$ in less than $112 \mu \mathrm{s}$. The output voltage settles to its new steady-state value of $5.82 \mathrm{~V}$ without oscillations. Hence, the simulation results show that the closed-loop system remains in stable condition in response to a peak voltage reference transient.

The effectiveness of the proposed SIDO converter to drive unbalanced load is also investigated. As an example, the first and second LED strings require an average current value of 80 and $30 \mathrm{~mA}$, respectively. Unlike the balanced load case with a constant peak inductor current limit, two distinct peak current limits are employed for unbalanced load such that two different average inductor (or load) current values can be generated in alternate clock cycles. Fig. 17 depicts the simulated steady-state waveforms from the SIDO buck LED driver with unbalanced load. The simulation results show that the first and second LED strings are regulated with an average current value of 80 and $30 \mathrm{~mA}$, respectively. For the first string, the simulated current ripple is $24.38 \%$ p-p and the output voltage ripple is $2.3 \%$ P-P. Also, for the second string, the simulated current ripple is $21.1 \%$ p-p and the output voltage ripple is $1.1 \%$ p-p. Either string meets the maximum ripple requirements. The simulation results demonstrate that the proposed SIDO converter is capable of delivering unequal currents to the two LED strings simultaneously.

\section{EXPERIMENTAL RESULTS}

The proposed SIDO buck LED driver was implemented on a field-programmable gate array (FPGA)-based hardware prototype in accordance with the design specification provided in Table I. The switching frequency is increased to $156.25 \mathrm{kHz}$ in order to satisfy the LED current ripple requirement. A photo of the experimental setup is shown in Fig. 18. The power stage of the buck converter consists of discrete ICs from International Rectifier such as power MOSFETs (IRF7828), dual-channel gate driver (IR2110), and output switches (IRF9388), as well as surface-mount inductor and low-ESR capacitors. In actual implementation, the top level of the proposed digital controller is partitioned into two major functional blocks. The functionality of the first block is to control the switching action of the power 


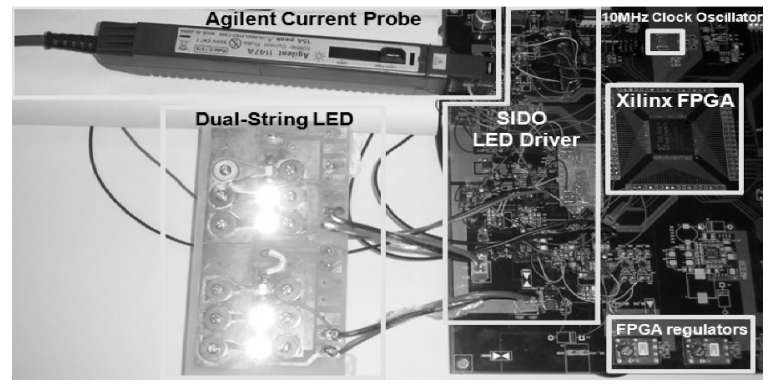

Fig. 18. Experimental setup for the proposed SIDO buck LED driver.

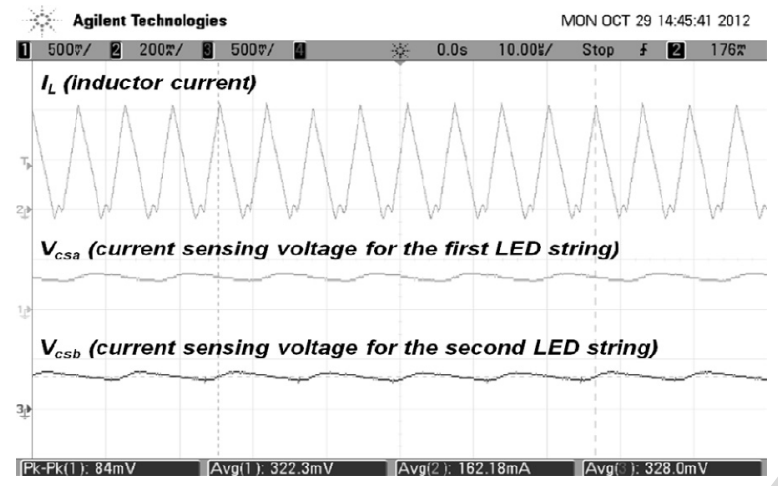

Fig. 19. Measured waveforms for inductor current and current sense feedback voltage.

stage by detecting the peak-crossing and zero-crossing events of the inductor current. It was implemented in hierarchical gatelevel schematics using primitives and macros available from the Xilinx Spartan-3 Generation library. Dead-time logic is included to prevent shoot-through current of the power switches. The second logical block is used to control the switching sequence of the two output switches by continuously monitoring the current-sense feedback signals. It was modeled as an FSM in Verilog RTL. Only one of the two output switches can be ON and the other must be OFF per switching cycle. Dead-time logic is also added to prevent cross conduction between outputs. The two logical blocks are synchronized by the system clock to ensure that the high-side power switch and the output switches are triggered from the same clock edge. The entire digital controller was implemented with Xilinx Spartan-3E (XC3S250E) FPGA. The quasi-hysteretic control logic was realized using 4-ns fast comparators (AD8611 from Analog Devices) and semicustom synchronous logic.

Using a current sensing resistor of $4 \Omega$ and reference voltage of $320 \mathrm{mV}$, the target current in each of the two LED strings is $80 \mathrm{~mA}$. Fig. 19 shows the current sensing feedback voltage $\left(V_{\mathrm{csa}}, V_{\mathrm{csb}}\right)$ from which the corresponding average load current can be obtained, i.e., $I_{\mathrm{LED}}=V_{c s} / R_{c s}$. The average inductor current is measured to be $162 \mathrm{~mA}$, which is the sum of the load currents in both LED strings. The average current values in the first and second LED string are measured to be around 80.6 and $82 \mathrm{~mA}$, respectively. The measured LED current ripple $\Delta i_{\mathrm{LED}}$ in either string is around $26 \%$ P-P, which is reasonably close to the simulated current ripple of $24 \%$ p-p. In addition, the nominal output voltages in the first and second string are

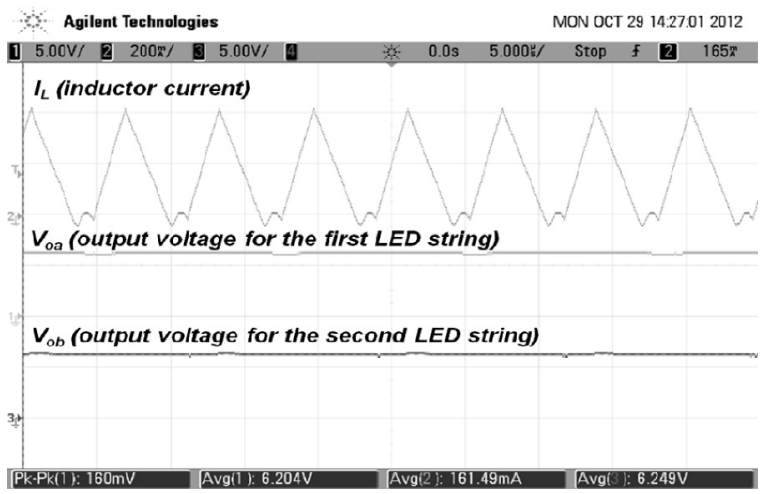

Fig. 20. Measured waveforms for inductor current and output voltage in either LED string.

measured to be 6.204 and $6.249 \mathrm{~V}$, respectively, as shown in Fig. 20. Under this balanced load condition, the measured current and voltage values are in close agreement between the two LED strings. The output voltage ripple is also measured to be around $2.57 \%$ p-P , compared to $2.3 \%$ p-p from simulation. Therefore, the experimental results are shown to be consistent with the corresponding simulation ones. On the other hand, the measured power conversion efficiency of the proposed SIDO converter is $80 \%$ which is comparable to conventional driving topologies [41]. The efficiency can be further increased by employing a current-sensing resistor with a smaller value.

The transient response of the proposed SIDO buck LED driver is verified experimentally by measuring its peak voltage reference step response. An 8-bit digital-to-analog converter (AD558 from Analog Devices) is used to enable programming of the peak voltage reference $V_{\text {peak }}$ and the current-sense voltage references $\left(V_{\mathrm{ref} a}, V_{\mathrm{ref} b}\right)$ by the Xilinx FPGA. The measured waveforms of the inductor current and the voltage at the current sensing nodes in response to a peak voltage reference step are shown in Fig. 21. The settling time of the transient response is also measured and compared with the simulated settling time. For the step-up response, it is observed that the current-sensing voltage $V_{\text {csa }}$ in the first LED string steps up from 81.8 to $325.4 \mathrm{mV}$, which corresponds to an increase in the average load current from 20.5 to $81.3 \mathrm{~mA}$. Similarly, the current-sensing voltage $V_{\mathrm{csb}}$ in the second LED string steps up from 94.1 to $327.6 \mathrm{mV}$, which corresponds to an increase in the average load current from 23.5 to $81.9 \mathrm{~mA}$. The settling time for the step-up response is measured to be $67 \mu \mathrm{s}$, compared to $63 \mu$ s from simulation. The measured results for the step-down response are the reverse of those from the step-up response. The only difference is that it takes longer for the step-down transient to settle. The settling time for the step-down response is measured to be $115 \mu \mathrm{s}$, compared to $112 \mu$ s from simulation. The measured settling times are shown to be very close to the simulated ones. The experimental results confirm that the system remains in stable condition when it is perturbed by the peak voltage reference transient.

The unbalanced load scenario in the proposed SIDO buck LED driver is also verified experimentally. The measured average load current values in the first and second LED string are 


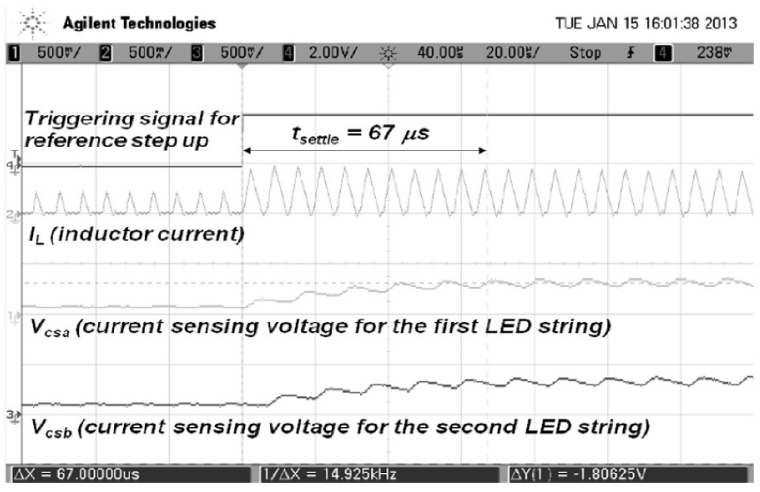

(a)

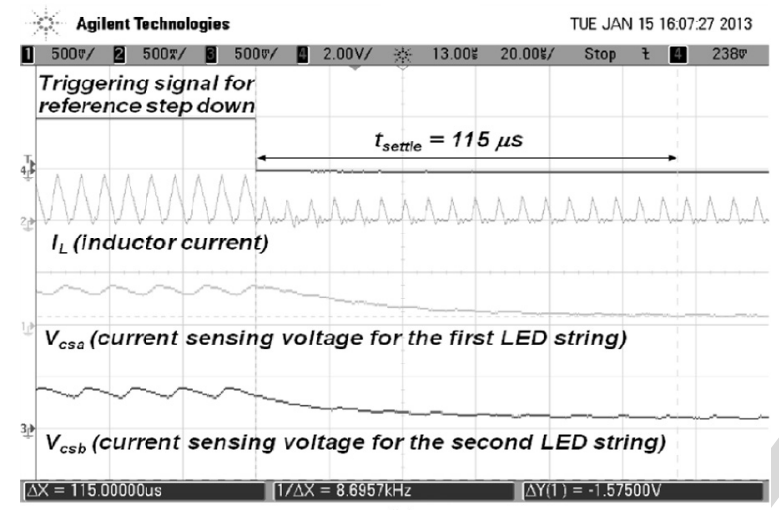

(b)

Fig. 21. Measured transient waveforms in response to (a) peak reference stepup and (b) peak reference step-down.

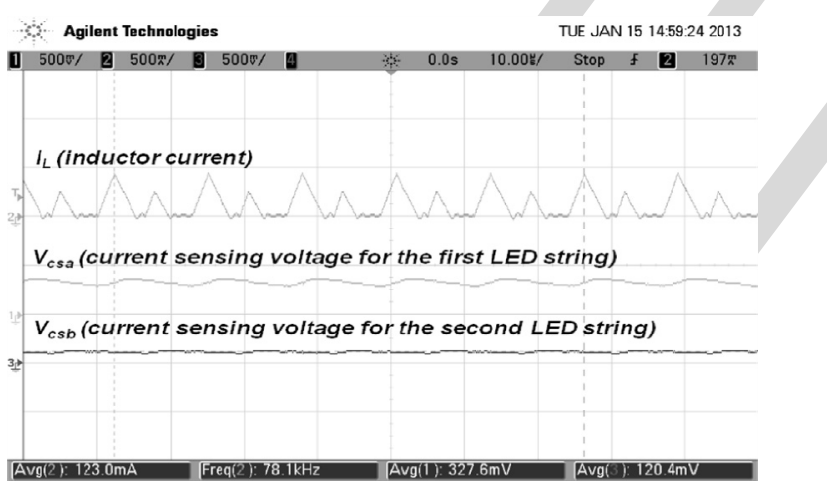

Fig. 22. Measured waveforms for inductor current and current sense voltages.

around 81.9 and $30.1 \mathrm{~mA}$, respectively. Fig. 22 shows the measured waveforms for the inductor current and the current-sensing voltage per string. The inductor current waveform indicates that the proposed driver operates in DCM with two distinct peak current limits. Fig. 23 shows the measured inductor current and the output voltage in either string. The measured output voltage values in the first and second LED string are 6.22 and $5.70 \mathrm{~V}$, respectively. The experimental results demonstrate that the proposed driver is capable of driving two independent LED strings concurrently with different load current.

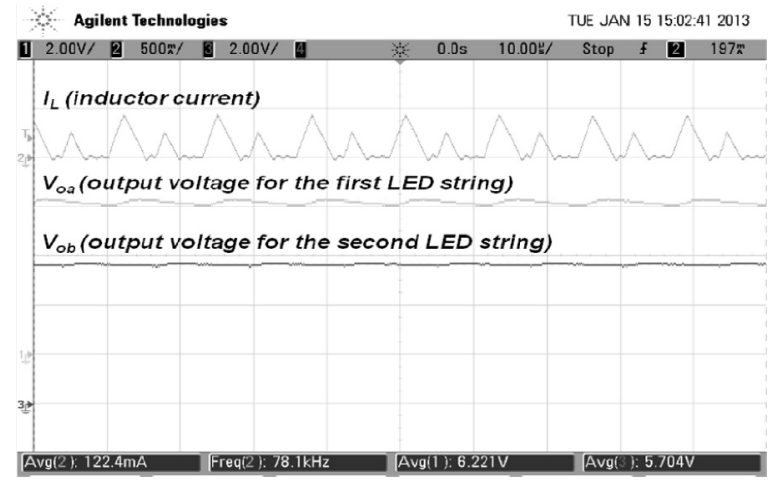

Fig. 23. Measured waveforms for inductor current and output voltages.

\section{CONCLUSION}

The proposed SIDO buck LED driver was implemented in 687 FPGA-based hardware. The experimental results correlate well 688 with simulation ones. The scalability of the proposed SIDO buck 689 LED driver to SIMO is closely examined. A general formula 690 for determining the theoretical maximum achievable number of 691 LED strings in SIMO is derived. The simulation results were 692 shown to be consistent with those obtained from the theoret- 693 ical model for the same design parameter values. The quasi- 694 hysteretic digital control scheme does not require loop com- 695 pensation which simplifies the control loop design and reduces 696 component count. In addition, the proposed SIMO architecture 697 offers the advantage of driving a larger number of parallel LED 698 strings without being limited by the maximum current rating of 699 the LED. It also enables dimming for the LED strings without 700 additional dimming transistors. Local bus voltage and current 701 optimization in each individual LED string compensates for the 702 variability of the LED's forward voltage, which reduces power 703 loss and enables mixing of white LEDs from different bins to 704 lower LED costs.

\section{REFERENCES}

[1] C.-Y Hsieh, C.-Y. Yang, and K.-H. Chen, "A charge-recycling buck-store and boost-restore (BSBR) technique with dual outputs for RGB LED backlight and flashlight module," IEEE Trans. Power Electron., vol. 24, no. 8, pp. 1914-1925, Aug. 2009.

[2] S.-Y. Lee, J.-W. Kwon, H.-S. Kim, M.-S. Choi, and K.-S. Byun, "New design and application of high efficiency LED driving system for RGBLED backlight in LCD display," in Proc. 37th IEEE Power Electron. Spec. Conf., 2006, pp. 1-5.

[3] H.-J. Chiu, Y.-K. Lo, J.-T. Chen, S.-J. Cheng, C.-Y. Lin, and S.-C. Mou, "A high-efficiency dimmable led driver for low-power lighting applications," IEEE Trans. Ind. Electron., vol. 57, no. 2, pp. 735-743, Feb. 2010.

[4] C.-H. Liu, C.-Y. Hsieh, Y.-C. Hsieh, T.-J. Tai, and K.-H. Chen, "SARControlled adaptive off-time technique without sensing resistor for achieving high efficiency and accuracy led lighting system," IEEE Trans. Circuits Syst. I, Reg. Papers, vol. 57, no. 6, pp. 1384-1394, Jun. 2010.

[5] C.-H. Wu and C.-L. Chen, "High-efficiency current-regulated charge pump for a white LED driver," IEEE Trans. Circuits Syst. II, Exp. Briefs, vol. 56 , no. 10, pp. 614-618, Oct. 2009.

[6] P. Malcovati, M. Belloni, F. Gozzini, C. Bazzani, and A. Baschirotto, "A $0.18 \mu \mathrm{m}$ CMOS $91 \%$-efficiency 0.1-to-2 A scalable buck-boost DC-DC converter for LED drivers," in Proc. ISSCC Dig. Tech. Papers, Feb. 2012 pp. $280-281$

[7] S. Rao, Q. Khan, S. Bang, D. Swank, A. Rao, W. Mclntyre, an P. K. Hanumolu, "A 1.2 A buck-boost LED driver with $13 \%$ efficiency 888 89

90

91

92

3

94

95

6

97

8

9

01

02

03
704


improvement using error-averaged SenseFET-Based current sensing," in Proc. ISSCC Dig. Tech. Papers, Feb. 2011, pp. 238-239.

[8] S.-I. Hong, J.-W Han, D.-H. Kim, and O.-K. Kwon, "A double-loop control LED backlight driver IC for medium-sized LEDs," in Proc. ISSCC Dig. Tech. Papers, Feb. 2010, pp. 116-117.

[9] M.-H. Huang and K.-H. Chen, "Single-inductor multi-output (SIMO) DC-DC converters with high light-load efficiency and minimized crossregulation for portable devices," IEEE J. Solid-State Circuits, vol. 44, no. 4, pp. 1099-1111, Apr. 2009.

10] E. Bonizzoni, F. Borghetti, P. Malcovati, F. Maloberti, and B. Niessen, "A $200 \mathrm{~mA} 93 \%$ peak efficiency single-inductor dual-output DC-DC buck converter," in Proc. IEEE ISSCC Dig. Tech. Papers, Feb. 2007, pp. 526619.

11] C--Y. Hsieh and K.-H. Chen, "Boost DC-DC converter with fast reference tracking (FRT) and charge-recycling (CR) techniques for high-efficiency and low-cost LED driver," IEEE J. Solid-State Circuits, vol. 44, no. 9 pp. 2568-2580, Sep. 2009.

[12] H. Chen, Y. Zhang, and D. Ma, "A SIMO parallel-string driver IC for dimmable LED backlighting with local bus voltage optimization and single time-shared regulation loop," IEEE Trans. Power Electron., vol. 27, no. 1, pp. 452-462, Jan. 2012.

13] D. Ma, W.-H. Ki, P. K. T. Mok, and C.-Y. Tsui, "Single-inductor multipleoutput switching converters with bipolar outputs," in Proc. IEEE Int. Symp. Circuits Syst., May 2001, vol. 3, pp. 301-304.

[14] D. Ma, W.-H. Ki, C.-Y. Tsui, and P. K. T. Mok, "A 1.8 V single-inductor dual-output switching converter for power reduction techniques," in Proc. IEEE Symp. VLSI Circuits, Jun. 2001, pp. 137-140.

[15] W.-H. Ki and D. Ma, "Single-inductor multiple-output switching converters," in Proc. 32nd IEEE Power Electron. Spec. Conf., Jun. 2001, vol. 1, pp. 226-231.

16] D. Ma, W.-H. Ki, C.-Y. Tsui, and P. K. T. Mok, "Single-inductor multipleoutput switching converters with time-multiplexing control in discontinuous conduction mode," IEEE J. Solid-State Circuits, vol. 38, no. 1, pp. 89-100, Jan. 2003

17] D. Ma, W.-H. Ki, and C.-Y. Tsui, "A pseudo-CCM/DCM SIMO switching converter with freewheel switching," IEEE J. Solid-State Circuits, vol. 38 no. 6, pp. 1007-1014, Jun. 2003.

[18] D. Kwon and G. A. Rincón-Mora, "Single-inductor multiple-output switching DC-DC converters," IEEE Trans. Circuits Syst. II, Exp. Briefs, vol. 56, no. 8, pp. 614-618, Aug. 2009.

[19] K.-S. Seol, Y.-J. Woo, G.-H. Cho, G.-H. Gho, and J.-W. Lee, "A synchronous multioutput step-up/down DC-DC converter with return current control," IEEE Trans. Circuits Syst. II, Exp. Briefs, vol. 56, no. 3, pp. 210214, Mar. 2009

[20] C.-W. Leng, C.-H. Yang, and C.-H. Tsai, "Digital PWM controller for SIDO switching converter with time-multiplexing scheme," in Proc. Int. Symp. VLSI Design Autom. Test, Apr. 2009, pp. 52-55.

21] Y.-J. Moon, Y.-S. Roh, J.-C. Gong, and C. Yoo, "Load-independent current control technique of a single-inductor multiple-output switching DC-DC converter," IEEE Trans. Circuits Syst. II, Exp. Briefs, vol. 59, no. 1, pp. 5054, Jan. 2012.

[22] F. Su, W.-H. Ki, and C.-Y. Tsui, "Ultra fast fixed-frequency hysteretic buck converter with maximum charging current control and adaptive for DVS applications," IEEE J. Solid-State Circuits, vol. 43, no. 4, pp. 815-822, Apr. 2008.

[23] W.-H. Ki, K.-M. Lai, and C. Zhan, "Charge balance analysis and state transition analysis of hysteretic voltage mode switching converters," IEEE Trans. Circuits Syst. I, Reg. Papers, vol. 58, no. 5, pp. 1142-1153, May 2011.

24] K. K.-S. Leung and H. S.-H. Chung, "Dynamic hysteresis band control of the buck converter with fast transient response," IEEE Trans. Circuits Syst. II, Exp. Briefs, vol. 52, no. 7, pp. 398-402, Jul. 2005.

[25] H. Eachempatti, S. Ganta, J. Silva-Martinez, and H. Martínez-Garcia, "SIDO buck converter with independent outputs," in Proc. 53rd IEEE Int. Midwest Symp. Circuits Syst., Aug. 2010, pp. 37-40.

[26] F. Su and W.-H. Ki, "Digitally assisted quasi-V² hysteretic buck converter with fixed frequency and without using Large-ESR capacitor," in Proc. ISSCC Dig. Tech. Papers, Feb. 2009, pp. 446-447.

[27] Cadence Spectre Circuit Simulator Datasheet [Online]. Available: http://www.cadence.com/products/cic/spectre_circuit/pages/default.aspx

[28] M. Doshi and R. Zane, "Digital architecture for driving large LED arrays with dynamic bus voltage regulation and phase shifted PWM," in Proc. Appl. Power Electron. Conf., Feb. 2007, pp. 287-293.

29] W.-S. Oh, D. Cho, K.-M. Cho, G.-W. Moon, B. Yang, and T. Jang, "A novel two-dimensional adaptive dimming technique of X-Y channel drivers for
LED backlight system in LCD TVs," J. Display Technol., vol. 5, no. 1, 806 pp. 20-26, Jan. 2009.

[30] Datasheet: LP8545, "LP8545 High-efficiency LED backlight driver for notebooks," Texas Instruments Incorporated, [Online] Available: http://www.ti.com/general/docs/lit/getliterature.tsp?genericPartNumber= lp8545\&fileType $=$ pdf

[31] Datasheet: MAX16814, "Integrated, 4-channel, high-brightness LED driver with high-voltage DC-DC controller,' Maxim Integrated Products, Inc., [Online], Available: http://datasheets.maximintegrated.com/ en/ds/MAX16814.pdf

[32] K. H. Loo, W. K. Lun, S. C. Tan, Y. M. Lai, and C. K. Tse, "On driving techniques for LEDs: Toward a generalized methodology," IEEE Trans. Power Electron., vol. 24, no. 12, pp. 2967-2976, Dec. 2009.

[33] W. K. Lun, K. H. Loo, S. C. Tan, Y. M. Lai, and C. K. Tse, "Bilevel current driving technique for LEDs," IEEE Trans. Power Electron., vol. 24, no. 12, pp. 2920-2932, Dec. 2009

[34] S. C. Tan, "General n-level driving approach for improving electrical-tooptical energy-conversion efficiency of fast-response saturable lighting devices," IEEE Trans. Ind. Electron., vol. 57, no. 4, pp. 1342-1353, Apr. 2010

[35] D. Ma and W.-H. Ki, "Fast transient PCCM switching converter with freewheel switching control," IEEE Trans. Circuits Syst. II, Exp. Briefs, vol. 54, no. 9, pp. 825-829, Sep. 2007.

[36] D. Ma, W.-H. Ki, and C.-Y. Tsui, "Single-inductor multiple-output switching converters in PCCM with freewheel switching," U.S. Patent 7432 614, Oct. 7, 2008

[37] Application Note: AN-1656, "Design challenges of switching LED drivers," Texas Instruments Incorporated, [Online] Available: http:// www.ti.com/general/docs/lit/getliterature.tsp?literatureNumber $=$ snva253 \&fileType $=$ pdf

[38] Solutions Guide, "LED drivers for high-brightness lighting," Texas Instruments Incorporated, [Online], Available: http://www.ti.com/ general/docs/lit/getliterature.tsp?baseLiteratureNumber $=$ snvy001

[39] R. W. Erickson and D. Maksimovic, Fundamentals of Power Electronics, 2nd ed. New York, NY, USA: Springer, 2001.

[40] Datasheet: SMD5730, "SMD 5730 White LED Datasheet," APT Electronics Ltd., [Online], Available: http://www.apt-hk.com/en/product/?95.html

[41] Z. Ye, F. Greenfeld, and Z. Liang, "Design considerations of a high power factor SEPIC converter for high brightness white LED lighting applications," in Proc. IEEE Power Electron. Spec. Conf., 2008, pp. 2657-2663.
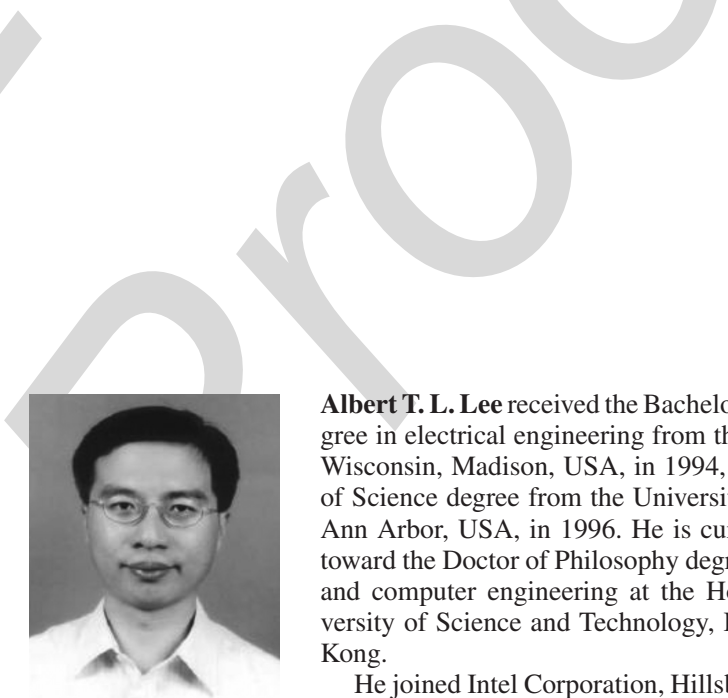

Albert T. L. Lee received the Bachelor of Science degree in electrical engineering from the University of 847 Wisconsin, Madison, USA, in 1994, and the Master 848 of Science degree from the University of Michigan, 849 Ann Arbor, USA, in 1996. He is currently working 850 toward the Doctor of Philosophy degree in electronic 851 and computer engineering at the Hong Kong Uni- 852 versity of Science and Technology, Kowloon, Hong 853 Kong.

He joined Intel Corporation, Hillsboro, OR, USA, in 1996 as a Senior Component Design Engineer and was involved in the development of Intel's P6 family microprocessors. In 2001, he served as a Senior Corporate Application Engineer in the System-Level Design Group at Synopsys Inc., Mountain View, CA, USA. In 2003, he joined the Hong Kong Applied Science and Technology Research Institute Company Ltd. and served as EDA Manager in the Wireline Communications Group. In 2006, he joined the Giant Electronics Limited as Hardware Design Manager and became Associate General Manager in 2008. His research interests include mixed-signal system-level design, LED driver, power management system, and very large scale integration circuits. is .

\section{政} 850 851
852 853 854 855 856 856
857 857
858 859 860 861 862 863 863
864 865 866 


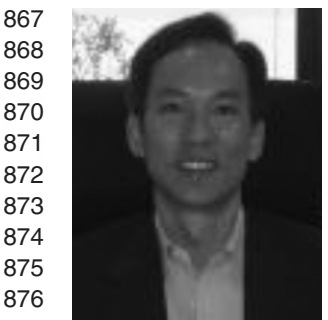

Johnny K. O. Sin (S'79-M'88-SM'96-F'12) reelectrical engineering from the University of Toronto, Toronto, ON, Canada, in 1981, 1983, and 1988, respectively.

From 1988 to 1991, he was a Senior Member of the research staff of Philips Laboratories, Briarcliff Manor, NY, USA. In August 1991, he joined the Department of Electronic and Computer Engineering, The Hong Kong University of Science and Technology, Kowloon, Hong Kong, where he has been a Full 8 Professor since 2001. He is the holder of 13 patents, and the author of more 879 than 270 papers in technical journals and refereed conference proceedings. His the IEEE Electron Devices Society. He is also a Technical Committee member of the International Symposium on Power Semiconductor Devices and IC's. He is a Fellow of the IEEE for contributions to the design and commercialization of power semiconductor devices. ceived the B.A.Sc., M.A.Sc., and Ph.D. degrees in

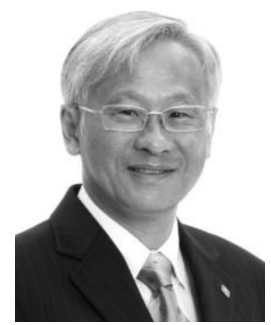

Philip C. H. Chan (SM'97-F'07) received the Bachelor of Science degree in electrical engineering from the University of California at Davis, Davis, USA, in 1973, and the Master of Science and Doctor of Philosophy degrees in electrical engineering from the University of Illinois at Urbana-Champaign, Urbana, USA, in 1975 and 1978, respectively.

He later joined Intel Corporation, Santa Clara, CA, USA, in 1981 and became a Senior Project Manager in Technology Development. He joined the Hong Kong University of Science and Technology (HKUST) in 1991 as a founding member. He served at HKUST as the Associate Dean of Engineering and Head of the Department of Electronic and Computer Engineering. He became the Dean of Engineering in September 2003 He joined the Hong Kong Polytechnic University, Hong Kong, in 2010 as the Deputy President and Provost. His research interests include very large scale integration devices, circuits, electronic packaging, and integrated sensors.

Dr. Chan received the ECE Distinguished Alumni Award from the University of Illinois, Urbana-Champaign in 2010. 
912 Q1. Author: Please provide year information in Refs. [27], [30], [31], [37], [38], and [40]. 